\title{
Mapping of the quasi-periodic oscillations at the flank magnetopause into the ionosphere
}

\author{
E. R. Dougal ${ }^{1,2}$, K. Nykyri ${ }^{2}$, and T. W. Moore $^{2}$ \\ ${ }^{1}$ Sandia National Laboratories, Albuquerque, NM, USA \\ ${ }^{2}$ Department of Physical Sciences, Embry-Riddle Aeronautical University, Daytona Beach, FL, USA
}

Correspondence to: E. R. Dougal (dougale@my.erau.edu)

Received: 24 April 2013 - Revised: 16 September 2013 - Accepted: 10 October 2013 - Published: 18 November 2013

\begin{abstract}
We have estimated the ionospheric location, area, and travel time of quasi-periodic oscillations originating from the magnetospheric flanks. This was accomplished by utilizing global and local MHD models and Tsyganenko semi-empirical magnetic field model on multiple published and four new cases believed to be caused by the KelvinHelmholtz Instability. Finally, we used auroral, magnetometer, and radar instruments to observe the ionospheric signatures. The ionospheric magnetic latitude determined using global MHD and Tsyganenko models ranged from 58.3-80.2 degrees in the Northern Hemisphere and -59.6 degrees to -83.4 degrees in the Southern Hemisphere. The ionospheric magnetic local time ranged between 5.0-13.8 $\mathrm{h}$ in the Northern Hemisphere and 1.3-11.9 h in the Southern Hemisphere. Typical Alfvén wave travel time from spacecraft location to the closest ionosphere ranged between $0.6-3.6 \mathrm{~min}$. The projected ionospheric size calculated at an altitude of $100 \mathrm{~km}$ ranged from $47-606 \mathrm{~km}$, the same order of magnitude as previously determined ionospheric signature sizes. Stationary and traveling convection vortices were observed in SuperDARN radar data in both hemispheres. The vortices were between $1000-1800 \mathrm{~km}$ in size. Some events were located within the ionospheric footprint ranges. Pc5 magnetic oscillations were observed in SuperMAG magnetometer data in both hemispheres. The oscillations had periods between 4 $10 \mathrm{~min}$ with amplitudes of 3-25 nT. They were located within the ionospheric footprint ranges. Some ground magnetometer data power spectral density peaked at frequencies within one tenth of a $\mathrm{mHz}$ of the peaks found in the corresponding Cluster data. These magnetometer observations were consistent with previously published results.
\end{abstract}

Keywords. Magnetospheric physics (Magnetopause, cusp, and boundary layers; Magnetosphere-ionosphere interactions; MHD waves and instabilities)

\section{Introduction}

Kelvin-Helmholtz instabilities (KHI) are important in explaining solar wind transport from the magnetosheath (MSH) into the magnetosphere (MSP), particularly during northward interplanetary magnetic field (IMF) (Otto and Fairfield, 2000; Fairfield et al., 2000; Nykyri and Otto, 2001; Hasegawa et al., 2004). Detection of southward IMF driven KHI events are possible, as discussed in Hwang et al. (2011). However, these conditions typically generate a more dynamic environment, causing irregular vortex signatures and evolutions at intermittent intervals, leaving preferential detection to cases driven by northern IMF. Their identification in spacecraft observations can still be challenging due to the vast size of the magnetosphere compared to spacecraft coverage. Determining a ground-based method of identification would therefore be an asset to the scientific community. To aid in this effort, the proper identification of an ionospheric signature is needed. This research is purposed to determine the ionospheric location, size and travel-time of a KHI occurring at the magnetospheric flanks. This will allow for the estimation of when and where to look in the ground based data to document a potential KHI signature in the ionosphere, as well as how large of a vortex to look for.

$\mathrm{KHI}$ is a phenomenon present at a boundary interface between two viscous fluids moving with different velocities. The onset condition for the KHI in magnetized plasma is 
given by the following relations

$\left[\boldsymbol{k} \cdot\left(\mathbf{V}_{1}-\mathbf{V}_{2}\right)^{2}\right]>\frac{n_{1}+n_{2}}{4 \pi m_{0} n_{1} n_{2}}\left[\left(\boldsymbol{k} \cdot \mathbf{B}_{1}\right)^{2}+\left(\boldsymbol{k} \cdot \mathbf{B}_{2}\right)^{2}\right]$,

with wave vector of the KH mode $\boldsymbol{k}$, number density $n$, shear flow velocity $\mathbf{V}$ and magnetic field $\mathbf{B}$. The subindices refer to the values at both sides of the shear flow boundary.

Based on 2-D MHD simulations constructed using initial conditions from Cluster observations, Nykyri et al. (2006) identified two locations within the KH wave where reconnection took place. Otto and Fairfield (2000) showed large and rapid magnetic field changes where the $B_{z}$ component of the magnetic field could assume an orientation not consistent with the field on both sides of the low-latitude boundary layer (LLBL). This can be explained by KHI if the $\boldsymbol{k}$ vector has a component along the $\mathbf{B}$ direction. MHD simulations of $\mathrm{KHI}$ indicate reconnection can occur inside the current layers generated by KHI, providing the major mass transport mechanism for solar wind entry into the MSP (Nykyri and Otto, 2001). Nykyri and Otto (2001) showed that B can be parallel at both sides of the boundary of the instability while the anti-parallel $\mathbf{B}$ is generated from the vortex motion of the KHI. A strongly twisted B can occur within multiple layers of the KHI wave, causing reconnection to occur inside the vortices, creating high-density magnetic islands. These formations can detach from the MSH, possibly explaining the observation of high densities and low temperatures of the plasma sheet. Hasegawa et al. (2009) identified signatures of local reconnection in a KHI current sheet, however due to its incipient nature, Hasegawa et al. (2009) believed this reconnection process was unlikely to lead to formation of the duskflank LLBL, but rather that the flank LLBL was a result from other mechanisms such as diffusion or remote reconnection unidentified by the Cluster spacecraft.

These vortices have been observed and simulated on other planets as well; KHI waves have been observed in Saturn's magnetopause (Masters et al., 2010) and multiple times in Mercury's magnetopause (Boardsen et al., 2010; Sundberg et al., 2010) by observing quasi-periodic plasma and magnetic field signatures of the spacecraft data during certain IMF conditions. KHI waves have also been produced in ionopause simulations of Venus using plasma parameters consistent with Venus spacecraft observations (Wolff et al., 1980; Terada et al., 2002).

Past studies have discussed the possible ionospheric effects of the KHI. These signatures were believed to be the effect of small-scale, field-aligned currents (FACs) which originated from the vortex generated by KHI. FACs can be generated by KHI as the vortex motion twists the magnetic field. Ampere's Law states a current will be produced in the direction of $\nabla \times \mathbf{B}$. In particular geometry, the vortex motion results where the $\nabla \times \mathbf{B}$ is aligned with the dominant magnetic field direction, thus creating a FAC. In addition, the reconnection process initiated by the KHI can create a parallel electric field and thus accelerate particles along the magnetic field line, creating a current aligned with the magnetic field.

Auroral bright spots were previously reported by Lui (1989) and Farrugia et al. (1994) as a potential consequence of the KHI activity. The dimensions of these auroral bright spots were observed to be 40 to $100 \mathrm{~km}$ (Lui, 1989) and 50 to $200 \mathrm{~km}$ (Farrugia et al., 1994). Lui (1989) and Farrugia et al. (1994) located these spots between approximately 78 degrees to 70 degrees and 77 degrees to 74 degrees magnetic latitude, and 14 to $16 \mathrm{~h}$ and $16 \mathrm{~h}$ in magnetic local time, respectively. Both findings' locations were thus consistent occurring in similar magnetic latitudes in the post-noon sector.

Traveling convection vortices are another ionospheric phenomena which may be produced by KHI (McHenry et al., 1990). McHenry et al. (1990) studied a chain of traveling convection vortices which he concluded were KHI induced. Radar observations from Sondrestrom showed that the path of the chain of vortices was along the convection reversal boundary and each vortex in the chain followed an alternating rotational direction pattern. These signatures, along with the lack of upstream solar wind pressure disturbances, eluded to McHenry et al.'s (1990) conclusion that this was probably a result of KHI activity in the MSP boundary region.

Low-frequency magnetic pulsations in the Pc5 range have been suggested as the effect of KHI when observed in the dawn region as studied by Ohtani et al. (1999). The Pc5 range lies between 1 and $10 \mathrm{mHz}$, having a period of 1.6 to $16 \mathrm{~min}$. Another cause of this signature could be an external pressure variation, however evidence of an observed dusk propagation of the wave with no compressional signature in the magnetic field data, and evidence that the wave traveled at a rate comparable to the MSH flow speed ruled out this other possibility. The Pc5 waves and polarizations of the rotation of the plasma flow velocity at the ground were consistent with the wave range and polarization in Geotail. Geotail traveled up to $1 \mathrm{~h}$ of magnetic local time and 6 degrees invariant latitude near the observing magnetometers. The ground station magnetometer oscillations observed were similar to the $5 \mathrm{~min}$ period observed in the Geotail data and were observed with an amplitude of a few $\mathrm{nT}$ per second with a peak power spectral density concurrent with the peak in the spacecraft data. The ground station amplitude range was approximately an order of magnitude less than the amplitude range of the spacecraft observations. The dominant ground magnetometer frequency was within two tenths of a $\mathrm{mHz}$ of the spacecraft's dominant magnetometer frequency.

The goal of this project is to determine the projected size, ionospheric location and the travel time of magnetospheric perturbations produced by KHI traveling from the magnetosphere to the ionosphere and to investigate possible ionospheric signatures from the ground, optical, and radar data. The projected size of the vortex in the ionosphere provides estimation of the size of the signature to look for in ground-based and spacecraft observations, such as auroral bright spots and traveling convection vortex sizes, 
respectively. Local MHD simulations were used to calculate the magnetospheric vortex size. The NASA Community Coordinated Modeling Center (CCMC) hosts global magnetospheric models, which provide an opportunity to map Earth's magnetic field lines from the observing spacecraft position to the ionosphere during the event's unique magnetospheric environment. Their models produce coordinates of the field line locations and magnetic field strengths every few hundred kilometers, which allows for the estimation of travel time from the perturbation to the ionosphere. Field line mapping was also performed using the Tsyganenko semi-empirical model, discussed in Sect. 3.2.

We chose this approach because modeling the KHI directly in the global MHD simulations is very difficult due to large system size and the fine numerical resolution required to resolve the magnetopause. In order to study the details of the KHI, the numerical diffusion of the code (which depends on the grid resolution) should be less than the diffusion produced by the KHI (less than $10^{9} \mathrm{~m}^{2} \mathrm{~s}^{-1}$ ). For example Fairfield et al. (2007) compared Geotail observations of the KHI during an extended period of northward IMF orientation with the BATS-R-US global model utilizing computationally expensive specialized $1 / 16 R_{E}$ resolution (not currently available in CCMC "runs on request" website). Despite this relatively high resolution, their simulation only produced linear waves that did not reach non-linear stage as observed in the Geotail data.

Other authors have studied KHI in global codes both during southward (Claudepierre et al., 2008; Hwang et al., 2011) and northward (Guo et al., 2010; Li et al., 2012) IMF orientations. These studies addressed the large-scale structure of the magnetopause oscillations, spectral power of oscillations (Claudepierre et al., 2008) and some were able to determine the phase speeds and wavelengths albeit using only a quartersystem and ignoring the effects of the M-I coupling (Li et al., 2012).

In the present work, we analyzed four new events of the KHI that occurred predominately during Parker-Spiral (PS) IMF orientation. Currently there are no previous works studying KHI in global codes during a PS and ortho-ParkerSpiral (OPS) IMF orientation. Studying the KHI during a PS and OPS IMF orientation in global MHD codes that include M-I coupling and that can simultaneously resolve the KHI at the flanks and high-latitude reconnection would be crucial in order to fully address the dawn-dusk asymmetries arising from asymmetric evolution of these processes and their mutual interaction. However, this study would require higher numerical resolution than currently available in CCMC "runs on request"-website.

We have organized the paper as follows: Sect. 2 describes Cluster and ionospheric instruments, event selection, global, local, and semi-empirical models, vortex size and perturbation travel time methodology; Sect. 3 describes the results of the field line mapping, perturbation travel times, and vortex sizes in the ionosphere and magnetosphere; Sect. 4 describes the ionospheric signatures observed and Sect. 5 concludes and discusses the findings.

\section{Methodology}

\subsection{Cluster and ionospheric instrument data}

We gathered data from two instruments on board Cluster using spin averaged $(4 \mathrm{~s})$ measurements. The magnetic field measurements are obtained from the Flux Gate Magnetometer (FGM) (Balogh et al., 1997, 2001) from all four spacecraft. Ion plasma measurements were obtained using the Cluster Ion Spectrometry (CIS) instruments (Rème et al., 2001). We make use of the temperature, velocity and density from the Hot Ion Analyser (HIA) on board spacecraft 1 and 3 . The proton velocity and densities for spacecraft 4 are obtained from the ion COmposition and DIstribution Function analyzer (CODIF).

The Imager for Magnetopause-to-Aurora Global Exploration (IMAGE) spacecraft and Polar spacecraft were used to study auroral ionospheric signatures. We utilized four of the five filters on the ultraviolet imager (UVI); atomic Oxygen 1304 and 1356, Lyman-Birge-Hopfield (LBH) short with a range from 140 to $160 \mathrm{~nm}$, and $\mathrm{LBH}$ long with a range from 160 to $175 \mathrm{~nm}$. Polar UVI has an angular resolution of 0.036 degrees, yielding a spatial resolution of about $11 \mathrm{~km}$, which could be able to resolve all auroral structures produced by KHI. IMAGE's far ultraviolet FUV imager has the capability to image in three wavelength regions: the Wideband Imaging Camera (WIC) in the $\mathrm{N}_{2}$ LBH bands in the 140 to $180 \mathrm{~nm}$ range, Spectrographic Imager (SI) 12 in the Doppler-shifted $\operatorname{Lyman}_{\alpha}$ emission around $121.8 \mathrm{~nm}$, and SI13 in a $5 \mathrm{~nm}$ passband centered around $135.6 \mathrm{~nm}$. IMAGE FUV has a spatial resolution of about $150 \mathrm{~km}$ (Bisikalo et al., 2003), which could be able to resolve some of the auroral bright spots generated by KHI.

SuperDARN was used to study radar signatures of KHI activity. It consists of a network of over thirty low-power, highfrequency radars to observe ionospheric plasma, located in both hemispheres, beginning in the mid-latitude range and extending to the polar regions. Each radar uses an array of phased antennae stepping in azimuth every 3.3 degrees, totaling a sector of $50 \mathrm{~km}$ and repeating this sector scan every 1 to $2 \mathrm{~min}$. SuperDARN has a resolution of about $45 \mathrm{~km}$ (Greenwald et al., 1995) beginning at $180 \mathrm{~km}$ from the radar, extending to a maximum range usually greater than $3500 \mathrm{~km}$ (Greenwald et al., 1995). SuperDARN's spatial and temporal resolution should be sufficient to resolve the ionospheric vortices.

SuperMAG, used to study the magnetic field signatures, is a network of over 200 ground-based magnetometers covering both hemispheres to provide magnetic field perturbations. SuperMAG offers 3-D vector measurements of the magnetic field, utilizing stations which provide absolute measurements 
Table 1. Event dates, times, and GSM location for Cluster spacecraft 1 (Events 1-7) and Geotail (Event 8).

\begin{tabular}{cccll}
\hline Event & Date & Event Time & MP Location $\left[R_{E}\right]$ & Reference \\
\hline 1 & $07 / 03 / 2001$ & $05: 00-06: 00$ & $-8.87,-16.62,4.11$ & Nykyri et al. (2006) \\
2 & $11 / 20 / 2001$ & $20: 15-20: 45$ & $-3.66,18.54,-2.63$ & Hasegawa et al. (2004) \\
3 & $06 / 06 / 2002$ & $13: 20-13: 50$ & $-3.53,-16.11,-5.62$ & Moore (2012) \\
4 & $06 / 13 / 2002$ & $15: 10-15: 19$ & $-5.27,16.21,5.40$ & Moore (2012) \\
5 & $06 / 19 / 2004$ & $08: 58-09: 22$ & $-6.25,-17.70,-2.62$ & Moore (2012) \\
6 & $06 / 19 / 2004$ & $09: 40-10: 00$ & $-6.35,-17.56,-2.76$ & Moore (2012) \\
7 & $07 / 28 / 2006$ & $03: 07-03: 26$ & $-13.08,-12.83,3.06$ & Hwang et al. (2011) \\
8 & $03 / 24 / 1995$ & $05: 30-06: 30$ & $-14.14,19.0,-0.26$ & Fairfield et al. (2000) \\
\hline
\end{tabular}

and others with relative measurements. SuperMAG has a temporal resolution of $1 \mathrm{~min}$, which should be sufficient for observing magnetic field perturbations generated by KHI (Gjerloev, 2009).

\subsection{Magnetospheric KHI event selection}

Table 1 displays the KHI event list used for this project, populated from previously published observations of KHI and events discovered by Moore (2012) from the Cluster data. Moore (2012) studied five years of Cluster data looking for quasi-periodic oscillations at the flank boundary for further study. These new events were modeled using our local MHD simulations utilizing four different magnetic field orientations with respect to shear flow velocity to test the impact of initial condition selection on KHI growth. Section 2.4 explains this in more detail. The events which proved to be KHI unstable in the local MHD simulations were used in this research. Most events chosen were observed by the European Space Agency's Cluster spacecraft constellation. Event 8 was observed using Geotail, a spacecraft launched by the Institute of Space and Astronautical Science and NASA. Table 1 documents the date, time, and geocentric solar magnetospheric (GSM) system location for each event for Cluster spacecraft 1 or Geotail. The spacecraft separation between the four Cluster spacecraft averaged were 1300, 90, 60, 400, 450, and $6000 \mathrm{~km}$ for Events 1-4, Events 5 and 6, and Event 7, respectively. All events exhibited signatures consistent with typical KHI behavior; quasi-periodic density, temperature, velocity, and magnetic field variations, alternating between typical MSH and MSP values. Rotating the Cluster data into boundary normal coordinates indicated that the normal component of the magnetic field showed a train of regular bipolar variations consistent with signatures observed in the local MHD simulations that were generated with each of the event's parameters. Event 7, occurring during southward IMF as discovered by Hwang et al. (2011), was added during the course of this research. Therefore, this particular case was only mapped into the ionosphere to determine its footprint location.

Figure 1 shows typical KHI signatures in the data from Event 3. The quasi-periodic nature of the plasma density, temperature, velocity, and magnetic field shows the spacecraft crossing from MSH-like plasma characterized by high number densities and low temperatures to MSP-like plasma that has typically low number densities and higher temperatures. When a KHI occurs, the perturbation can twist the magnetopause as illustrated in Fig. 2. As the wave passes by the spacecraft, evidence of both MSP and MSH-like plasma become present in the time series data sequentially.

\subsection{Global magnetospheric models}

\subsubsection{Global MHD models}

CCMC hosts multiple global MHD models for community use, including four models which provide a magnetic field line tracing capability; Open Geospace General Circulation Model (OpenGGCM), Block-Adaptive-Tree-SolarwindRoe-Upwind-Scheme (BATS-R-US) model, Global Solar Wind-Magnetosphere-Ionosphere Coupling (GUMICS) model, and Lyon-Fedder-Mobarry (LFM) model. After consulting with CCMC personnel, it was recommended to use OpenGGCM and BATS-R-US for our research needs. Figure 3 displays two of CCMC's global models and their mapped field lines for Event 3 at 13:20 UT. The two left figures show the field lines in the $Y X$ and $Z Y$ frame from the OpenGGCM model, and the two right figures show identical plots from the BATS-R-US model. Comparing the figures, the OpenGGCM and BATS-R-US field lines have a difference of approximately $[2,2,3] R_{E}$ in the $x, y, z$ direction, respectively.

The minor differences in the results between OpenGGCM and BATS-R-US are likely due to the updating of the dipole moment and the differences in their numerical scheme when solving the MHD equations. As both models utilize input coordinates in different systems, all values shown in this paper will reflect the model's unique coordinate system. BATSR-US solves the 3-D MHD equations using a numerical scheme related to Roe's Approximate Riemann Solver. This solver allows for a simulation parameter to be set to update the dipole moment with time and is solved on a finite volume adaptive grid (Powell et al., 1999; Gombosi et al., 2002, 2004; Tóth et al., 2012). BATS-R-US utilizes a 2-D 

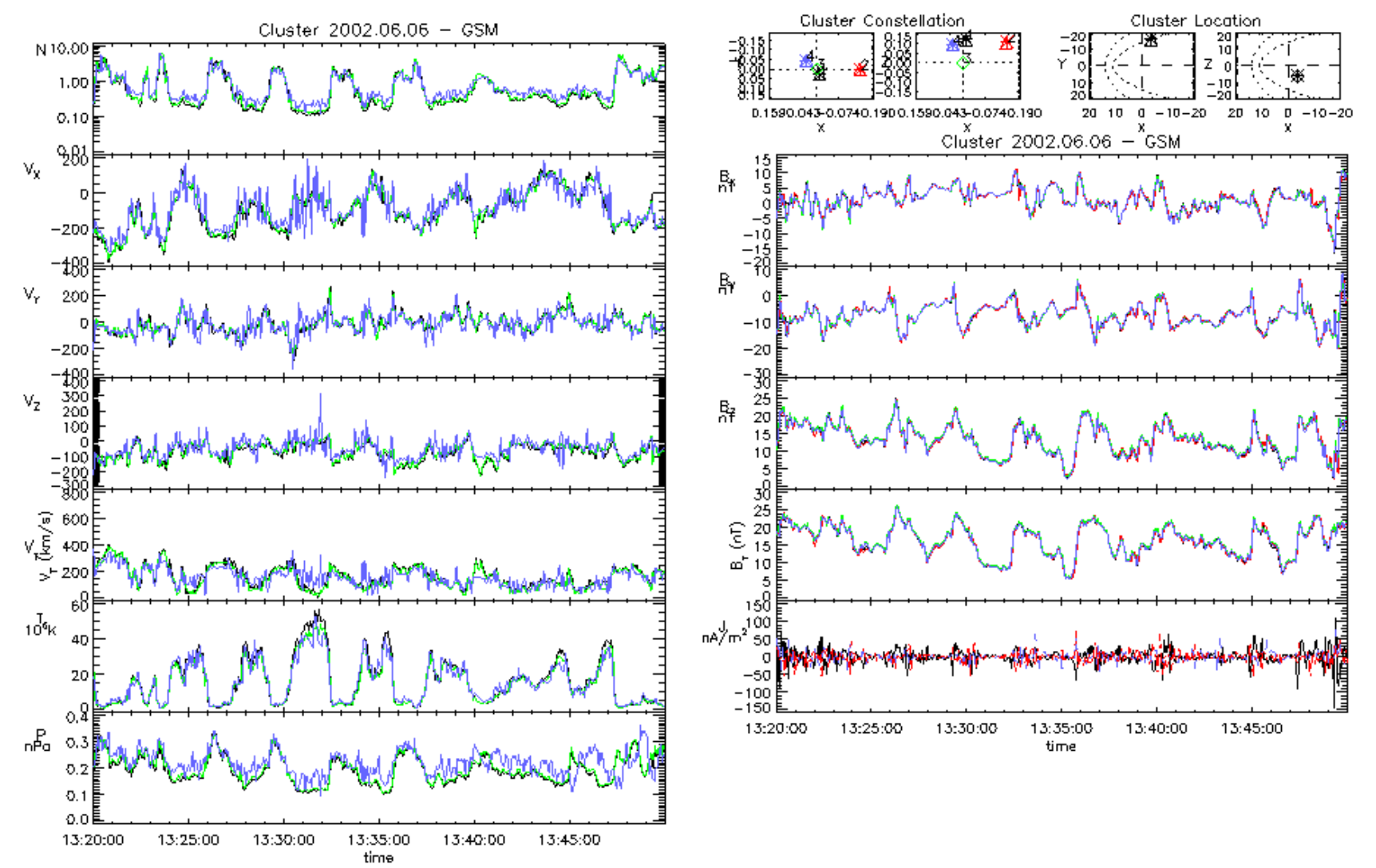

Fig. 1. Cluster plasma (left) and magnetic field (right) observations for Event 3 in GSM coordinates, which was used to determine the MSH and MSP values for the local MHD model. In order, the four spacecrafts are represented by black, red, green, and blue colors. The left panel, from the top, displays ion density, three velocity components, total velocity, plasma temperature and pressure. The right panel, from the top, displays three magnetic field components, total magnetic field, and current density. The four smaller panels on the top right hand corner show the Cluster constellation and location with the asterisk and diamond representing the beginning and ending of the interval, respectively.

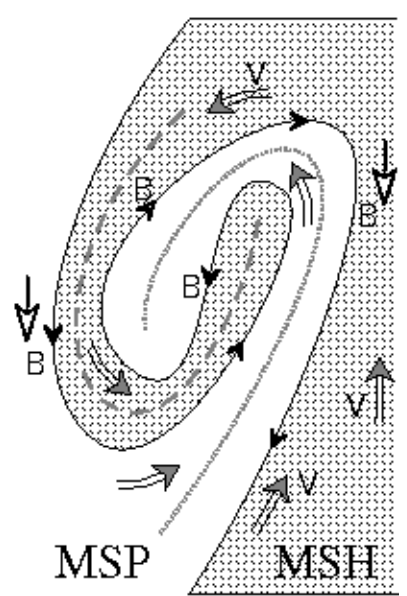

Fig. 2. Illustration taken from Nykyri and Otto (2001) of a KHI vortex causing mixing at the MSP/MSH boundary layer. electrodynamic potential solver to model the near-Earth environment. OpenGGCM solves the resistive MHD equations using second order explicit time integration with conservative and flux-limited spatial finite differences and is coupled with the Coupled Thermosphere Ionosphere Model for near-Earth approximations, a 3-D electric potential solver. Both programs use a dipole approximation to generate the ionospheric footprint from the end of their prospective ionospheric solvers to the Earth's surface. OpenGGCM does not update its dipole moment with time throughout the simulation. It uses a stretched Cartesian grid and does not include energetic particle drifts and ring current physics (Raeder et al., 2001). As BATS-R-US has the ability to couple with a ring current model, we chose to exclude these physics to stay consistent with the capabilities of OpenGGCM. Considerations for the coupling of a ring current model are discussed further in Sect. 3.1. We ran two simulations for each event and model, one at the start of the event time window and the other at then end of the event time window. The approximate ending altitude for all events using OpenGGCM was $3.7 R_{E}$ and 3.5 $R_{E}$ for BATS-R-US, however the field line mapping 

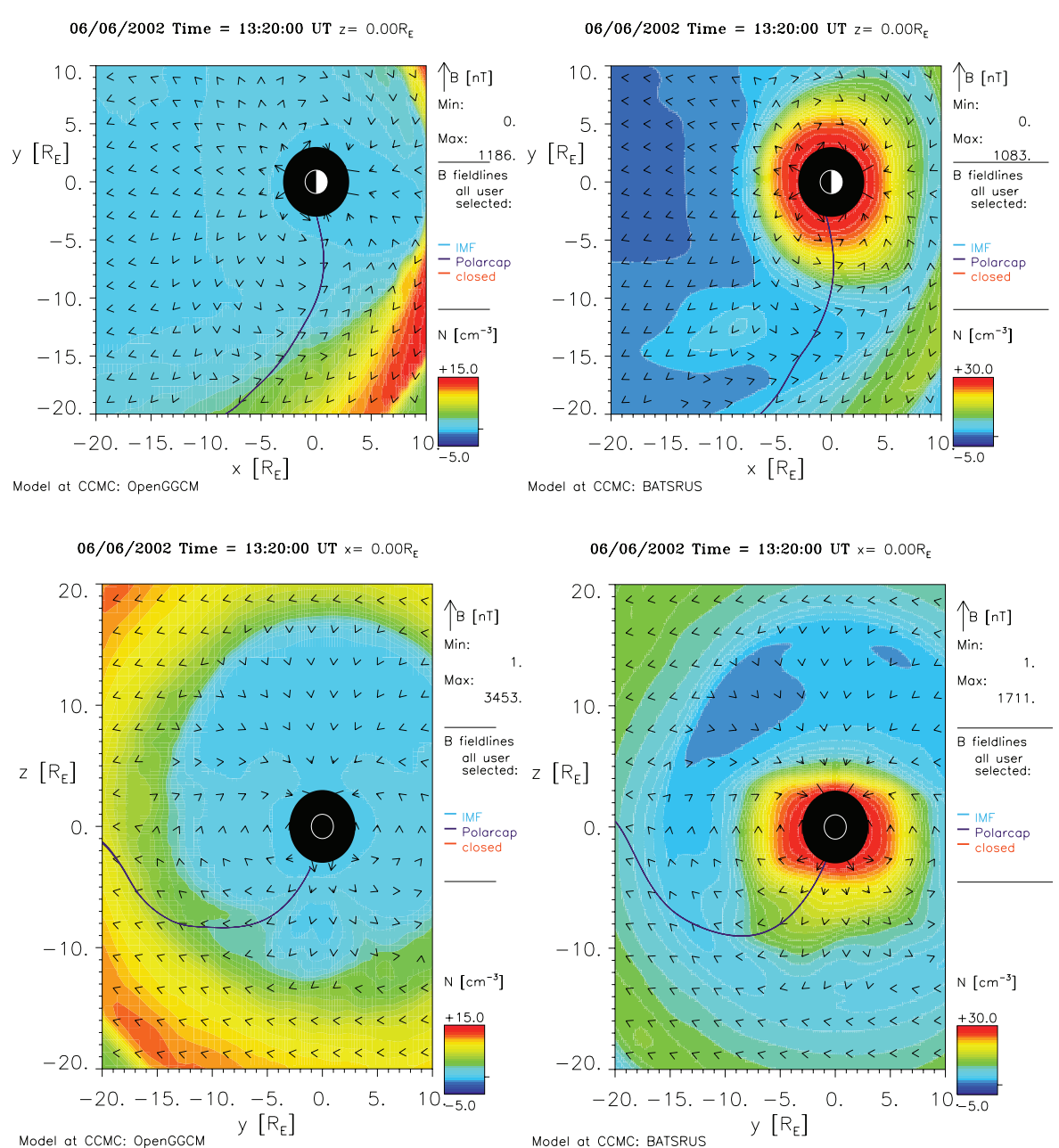

Fig. 3. Mapped field line positions of Event 3 at 13:20 UT for OpenGGCM and BATS-R-US. The top- and bottom-left images were produced from OpenGGCM and the top- and bottom-right images were produced from BATS-R-US. The spacecraft coordinates can be references from Table 1. Each panel illustrates the magnetic field direction using a black arrow and density using the color bar.

capability projects the ionospheric footprint location down to the Earth's surface following a field line generated by dipole approximation. The final coordinates at the Earth's surface, were expressed in the same coordinate system, magnetic latitude and magnetic local time.

\subsubsection{Tsyganenko 96 model}

A third model, Tsyganenko 96 (TS96) model, was additionally utilized as it is traditionally used for field line mapping purposes between ionosphere and magnetosphere, such as in Wing et al. (2005). TS96 is a semi-empirical approximation of the global magnetospheric magnetic field (Tsyganenko and Stern, 1996). Observations from a variety of spacecraft are combined with major external magnetospheric sources to represent the magnetic environment. The TS96 version of the model includes a defined magnetopause, Region 1 and 2 Birkeland current systems, and IMF boundary penetration. Because of the empirical nature of this model, it inherently includes kinetic physics unlike the global MHD models. The mapped field lines were calculated using GEOPACK2008 (Tsyganenko et al., 2008) which includes an external TS96 model and internal International Geomagnetic Reference Field (IGRF) Model Version 11.0 (Finlay et al., 2010). The field lines were mapped from Earth to the magnetosphere location at a resolution of 0.05 degrees of latitude and 1.0 degrees of longitude to find which field lines came within $0.3 R_{E}$ of our spacecraft location. This corresponds to approximately $56 \mathrm{~km}$ of latitude and $19 \mathrm{~km}$ of longitude resolution, respectively, taken at 70 degrees latitude. This was performed at the start and end of the event time window for the location of Cluster spacecraft 1 for Events 1-7 and Geotail for Event 8.

\subsection{Local 2-D MHD simulations}

The MHD simulations use a computational technique to replace the partial differential equations with systems of 
algebraic equations to provide a numerical solution (Nykyri, 2003). The resistive MHD equations are used in the simulations (Otto, 1990) and are solved using a finite difference leap frog scheme (Potter, 1973). The simulation-initial conditions were determined by the following equations (Nykyri et al., 2006):

$$
\begin{aligned}
& B_{x 0}=\frac{\mathbf{B} \cdot \boldsymbol{v}}{v} \\
& B_{z 0}^{2}=B^{2}-B_{x 0}^{2} \\
& B_{y 0}(x)=0 \\
& v_{x 0}(x)=v_{0}(x) \\
& v_{y 0}(x)=0 \\
& v_{z 0}(x)=0 \\
& \alpha=\arccos \left(B_{z 0} / B_{\mathrm{MSP}}\right) \\
& \beta=\arccos \left(B_{z 0} / B_{\mathrm{MSH}}\right),
\end{aligned}
$$

with $\alpha$ and $\beta$ angles between the magnetic field at either side of the boundary, and with respect to direction, perpendicular to shear flow plane where the $x$ component is aligned with the MSH flow and $z$ component is perpendicular to the flow. All quantities are normalized to the characteristic values for the system with length scales $l$ to typical length $L_{0}$; density $\rho$ to $\rho_{0}=n_{0} m_{0}$ with number density $n_{0}$ and ion mass $m_{0}$; magnetic field $\mathbf{B}$ to $B_{0}$; velocity $\boldsymbol{v}$ to typical Alfvén velocity $v_{\mathrm{a}}=B_{0} / \sqrt{\left(\mu_{0} \rho_{0}\right)}$; pressure $P$ to $P_{0}=B_{0}^{2} /\left(\mu_{0}\right)$; current density $J_{0}=B_{0} /\left(\mu_{0} L\right)$; and time $t$ to characteristic Alfvén transit time $\tau_{\mathrm{a}}=L_{0} / V_{\mathrm{a}}$. The simulations were developed in a magnetospheric inertial frame. The typical length $L_{0}$ is normalized to the approximate magnetopause thickness at the source region of the KHI.

In order to study the evolution of the fastest growing wave mode, the simulation box length, $x$, was adjusted to a wavelength, $\lambda=4 \pi a$, according to Miura and Pritchett (1982), where $a$ is the velocity shear layer thickness, $a=3 L_{0}$. The appropriate $L_{0}$ for the simulation of each event was computed from the observed wave length, $\lambda=v_{\mathrm{ph}} T$, estimated by Cluster measurements of the phase velocity, $v_{\text {ph }}$ and the wave period, $T$. The simulation dimensions were therefore adjusted to $[x, y]=[40,80] L_{0}$, where the larger system size in $y$ was chosen to minimize the effect of boundary conditions (such as reflection of waves) to the evolution of KHI at the center of the simulation box. The boundary conditions are periodic in $x$ and reflective in $y$ dimension and uses an adjustable grid of $403 \times 403$ grid points and a maximum resolution of 0.1 ( 10 grid points per $\left.L_{0}\right)$ around the velocity shear layer. $L_{0}$ was approximated to $1000 \mathrm{~km}$ for Event 1 and $600 \mathrm{~km}$ for Events 2-6.

The phase speed, $v_{\text {ph }}$, was estimated using two different methods: (1) $v_{\mathrm{ph}} \approx \frac{1}{2} v_{\mathrm{MSH}}$ (Miura and Pritchett, 1982), where $v_{\mathrm{MSH}}$ is the magnitude of the magnetosheath plasma velocity observed by Cluster, and (2) $v_{\mathrm{ph}} \approx v_{\mathrm{HT}}$, where $v_{\mathrm{HT}}$ is the de Hoffman Teller (HT) frame velocity (Sonnerup et al., 1995). The HT frame is a frame where the convec-
Table 2. Orientation of the magnetic field geometry for Events 3-6 using the notation shown in Fig. 4.

\begin{tabular}{ccc}
\hline Event & MSH & MSP \\
\hline 3 & 1 & B \\
4 & 2 & A \\
5 & 1 & B \\
6 & 1 & B \\
\hline
\end{tabular}

tion electric field vanishes, thus indicating an approximately steady-state plasma configuration. The $\mathrm{HT}$ velocity, $v_{\mathrm{HT}}$, is determined by minimizing $\left|\left(\boldsymbol{v}-\boldsymbol{v}_{\text {obs }}\right) \times B_{\text {obs }}\right|^{2}$ in terms of the constant transformation velocity $\boldsymbol{v}$ for a given data set (Sonnerup et al., 1995).

The simulation magnetic field component $B_{x}$ is calculated by projecting the observed magnetic fields on both sides of the boundary along the MSH velocity vector (Eq. 2). The magnetic field vector perpendicular to the shear flow plane, $B_{z}$, is also calculated from Eq. (2). The initial density, pressure, velocity, and magnetic field magnitudes are calculated using hyperbolic tangent profiles shown by Otto and Fairfield (2000). Because we are using 2-D simulations and the real magnetosphere is 3-D, where the $\boldsymbol{k}$ vector of the wave mode is not restricted to the equatorial plane, but will propagate along the direction where the ratio between the shear flow and the Alfvén speed is maximized (Nykyri et al., 2006), we tilted the shear flow plane at various angles to see whether an unstable boundary could occur. Using the angle with the best case to result in a KHI unstable boundary, Event 3 was tilted $10^{\circ}$, Event 4 was tilted $35^{\circ}$, and Events 5 and 6 were tilted $15^{\circ}$. Figure 4 illustrates the possible MSH and MSP magnetic field orientations of each simulation. Because the Cluster observations were already of the perturbed boundary, we ran four simulations for each event to observe the effect of the sign of the $B_{x}$ component with respect to the shear flow. The case chosen for further research was determined by correlating the simulation case conditions with the Cluster data as well as with the boundary layer structure obtained from the global MHD models. Case 1 set the MSH $B_{x}$ orientation as anti-parallel and MSP $B_{x}$ orientation as parallel, Case 2 set the MSH $B_{x}$ orientation as parallel and MSP $\mathbf{B}_{x}$ orientation as anti-parallel, Case 3 set both MSH and MSP $B_{x}$ orientation as anti-parallel and Case 4 set both MSH and MSP $B_{x}$ orientation to parallel. For these five events, the $B_{z}$ component was positive on both sides of the boundary. Event 3 was orientated like Case 4, Event 4 was orientated like Case 2, and Events 5 and 6 were orientated like Case 1. The final geometry used for each event is documented in Table 2 using the nomenclature from Fig. 4.

In order to compare the simulation and observation, a virtual spacecraft was inserted into the MHD simulation. The local 2-D simulation results, shown on the right windows in Figs. 5 and 6, were then compared to the boundary normal 

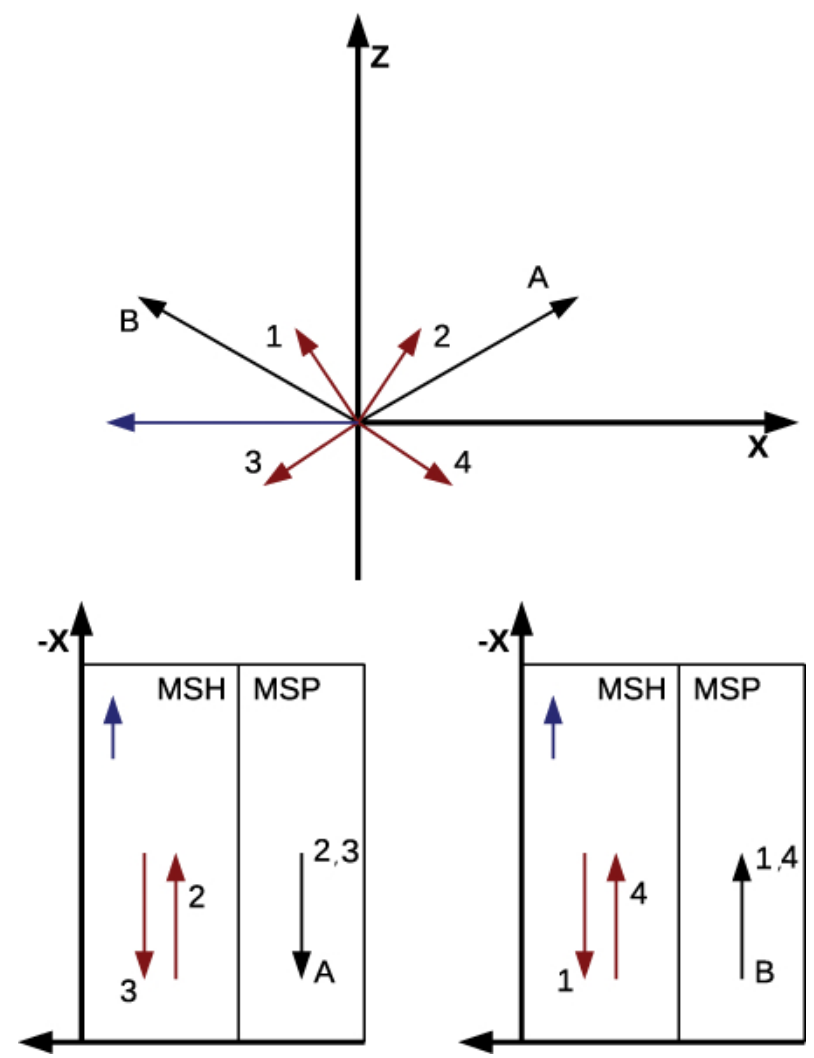

Fig. 4. The top figure shows the possible orientation of the MSP (black arrow) and MSH (red arrow) magnetic field. The bottom figure shows the four possible orientations of the local MHD plot output as seen by the user; Case 1 set the MSH $B_{x}$ orientation as anti-parallel and MSP $B_{x}$ orientation as parallel with respect to the $V_{\mathrm{MSH}}$, Case 2 set the MSH $B_{x}$ orientation as parallel and MSP $B_{x}$ orientation as anti-parallel,with respect to the $V_{\mathrm{MSH}}$, Case 3 set both MSH and MSP $B_{x}$ orientation as anti-parallel with respect to the $V_{\mathrm{MSH}}$, and Case 4 set both MSH and MSP $B_{x}$ orientation to parallel with respect to the $V_{\mathrm{MSH}}$. The red arrows, numbered 1-4, represent each case. The blue arrow represents the $V_{\mathrm{MSH}}$ flow. The black arrows, labeled $\mathrm{A}$ and $\mathrm{B}$, represent the two possible orien-

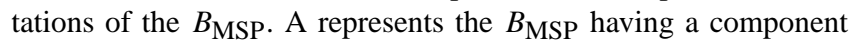
directed toward the Earth and B represents the $B_{\mathrm{MSP}}$ flow having a component directed tailward. Table 2 documents the orientations for Events 3-6.

coordinate Cluster data shown on the left windows of Figs. 5 and 6. Comparing the peak and trough values for number density and temperature to the variables during the event, the simulation values correspond with the observed values. The sharp transition between the number density and temperature in the simulation replicates the quasi-periodic signature as seen in the observations. The bipolar variation of the normal component of the magnetic field $\left(B_{y}\right)$ also indicates the presence of a wave at the boundary, corresponding to the maximum variance direction $(J)$ in the Cluster boundary normal data.

\subsection{Determining the ionospheric vorticity area}

The frozen-flux theorem was the basis for determining the ionospheric vortex size

$\phi_{\mathrm{M}}=\phi_{\mathrm{I}}$,

where $\phi=B A$ is the magnetic flux and subindices " $\mathrm{M}$ " and "I" refer to the magnetosphere and ionosphere, respectively. The ratio of the magnetic fields for the two regions is calculated to determine the size of the projected ionospheric vortex,

$A_{\mathrm{I}}=A_{\mathrm{M}} \frac{B_{\mathrm{M}}}{B_{\mathrm{I}}}$,

where $B_{\mathrm{M}}$ is the average value of $B_{\mathrm{MSP}}$ and $B_{\mathrm{MSH}}, B_{\mathrm{I}}$ is determined from the IGRF, $A_{\mathrm{I}}$ is the projection ionospheric area and $A_{\mathrm{M}}$ is the magnetospheric area of the vortex determined from local MHD simulations.

Figure 7 shows the simulation onset and growth of the KHI vortex during Event 3. The top- and bottom-left figures show the velocity vectors represented by the arrows and magnetic field strength depicted by color. The top- and bottom-right figures show the density represented by color. The black lines are magnetic field lines projected onto shear flow plane. The two top figures show the onset of the vortex and the bottom two figures represent how the vortex has evolved over time. Fluid elements, represented by asterisks, were initialized at the onset of the simulation at the MSH/MSP boundary. There were no initial velocity vectors on the MSP side as the area estimates were done in the MSP inertial frame to check whether the fluid elements indeed made a vortex structure that could produce a field aligned current. The plasma fluid element's location during the simulation was integrated from the plasma velocity. Each window shows a simulation geometry spun 180 degrees from what was illustrated in Fig. 4. In the simulation, the MSP region can be identified by its low density value. Once the vortex fully developed, we measured the dimensions where the plasma fluid elements created a full rotation within the vortex. In Fig. 7, the full rotation of plasma fluid elements centered around $[9,-2]$ in $X Y$ normalized units, respectively. When $A_{\mathrm{M}}$ was calculated from the simulation, new vortex dimensions for the ionospheric vortex $A_{\mathrm{I}}$ were approximated from Eq. (11) by conserving the ratio of the magnetospheric area dimensions.

\subsection{Perturbation travel time}

Kinematics was used to determine the amount of time it would take for the perturbation originating from the magnetosphere to travel to the ionosphere along magnetic field lines. In order to calculate the time lag between a KHI occurrence and a potential ionospheric signature, the Alfvén speed was calculated using the average magnetic field strength 

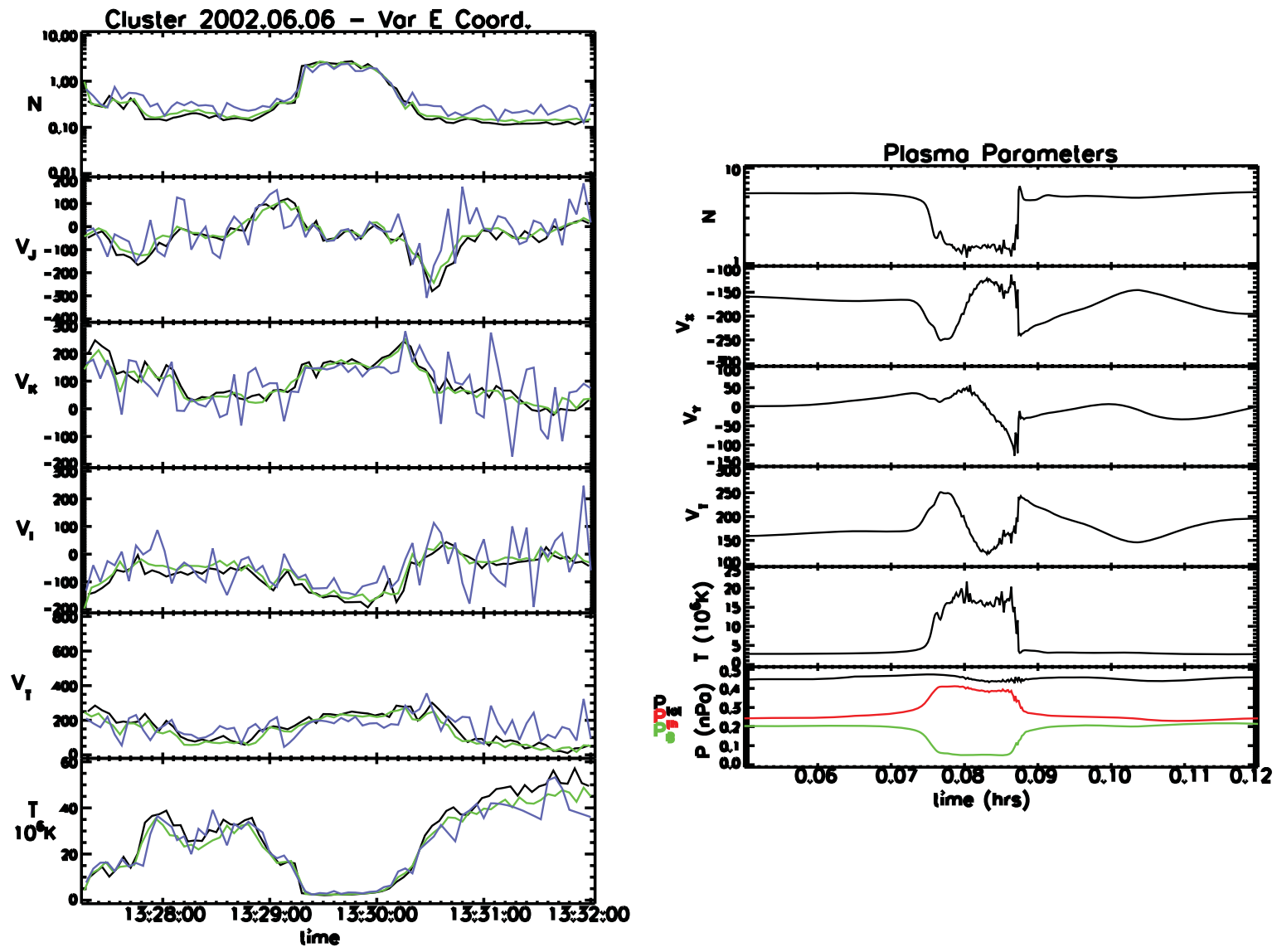

Fig. 5. Cluster and simulation plasma data for Event 3. The windows on the left display Cluster data in boundary-normal coordinates, used to determine the MSH and MSP values for the local MHD model. In order, the four spacecrafts are represented by black, red, green, and blue colors. From the top, the plot displays number density, components of velocity and total velocity and temperature. The windows on the right display the MHD simulation data. From the top, the plot displays number density, components of velocity and total velocity, temperature and components of pressure.

associated with a given field line position.

$\mathrm{d} r=\left|\boldsymbol{r}_{2}-\boldsymbol{r}_{1}\right|$

$V_{\mathrm{a}_{\mathrm{avg}}}=\frac{V_{\mathrm{a}_{1}}+V_{\mathrm{a}_{2}}}{2}$

$\delta t=\frac{\mathrm{d} r}{V_{\mathrm{a} a v g}}$

with Alfven speed $V_{\mathrm{a}}=\frac{B}{\sqrt{\mu \rho}}$ and subindices which refer to the different positions of the given magnetic field line. The difference in vector position between two points along a field line, $\mathrm{d} r$, was calculated. Figure 8 illustrates this method; the Alfvén speed was averaged between these two points, then divided under $\mathrm{d} r$ to determine the length of time it took to travel from $\boldsymbol{r}_{1}$ to $\boldsymbol{r}_{2}$. This calculation occurred at each point along the field line, allowing the change in time $\delta t$ to be accumulated over the entire length of the field line. All magnetospheric variables needed for this calculation were taken from the CCMC model variables at each respective location.
The ending altitude for this analysis was $3.6 R_{E}$, the average termination altitude for the global MHD models. However, our altitude of interest in the ionosphere was $100 \mathrm{~km}$, as it is the average auroral altitude (Deehr et al., 2005) and we are interested in looking at auroral data for potential optical signatures. Convection vortices, another manifestation of potential signatures, can be observed by the Super Dual Auroral Radar Network (SuperDARN) which looks at reflections in the $F$ region (150 to $800 \mathrm{~km}$ ) (Greenwald et al., 1995). The change in Alfvén speed during the descent to Earth from the models termination altitude into the ionosphere was studied to determine its effect on our travel time results. However, after considering changes such as ion density and magnetic field strength, we determined the additional fractions of a second were negligible. 

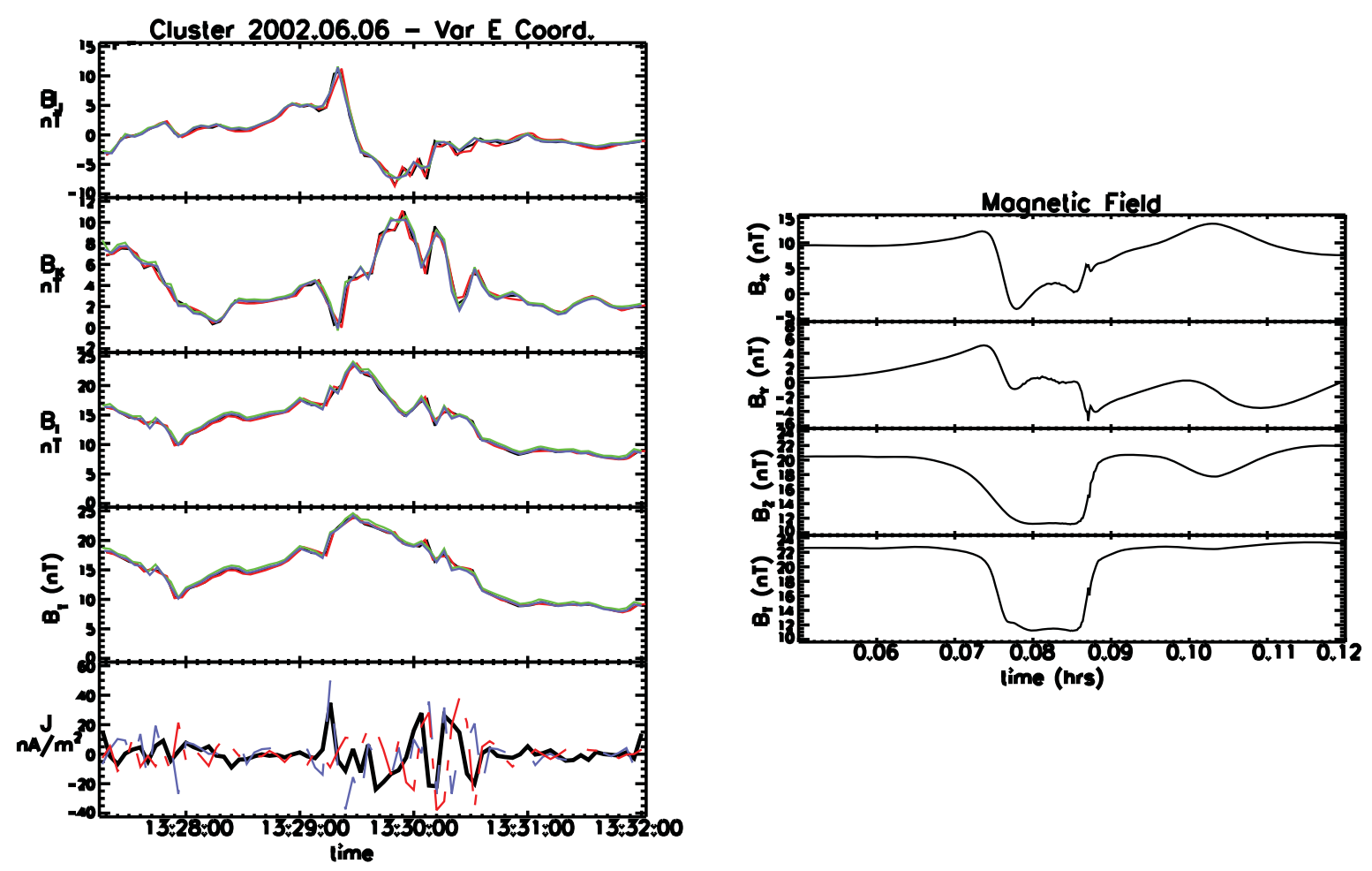

Fig. 6. Cluster and simulation magnetic field data for Event 3. The windows on the left display Cluster data in boundary-normal coordinates, used to determine the MSH and MSP values for the local MHD model. In order, the four spacecrafts are represented by black, red, green, and blue colors. From the top, the plot displays components of magnetic field ( $B_{J}$ is the maximum variance (normal), $B_{K}$ is the intermediate variance, and $B_{I}$ is the minimum variance (tangential) component), total magnetic field, current density. The windows on the right display the MHD simulation data. From the top, the plot displays components of magnetic field and total magnetic field.

Table 3. Average ionospheric footprint locations in magnetic latitude and local time (UT) coordinates. The subindices refer to the mapped field line locations ending in the northern $(\mathrm{N})$ or southern (S) pole.

\begin{tabular}{ccccc}
\hline Event & MLAT $_{\mathrm{N}}$ & MLT $_{\mathrm{N}}$ & MLAT $_{\mathrm{S}}$ & MLT $_{\mathrm{S}}$ \\
\hline 1 & 66.4 & 9.1 & -71.9 & 3.5 \\
2 & - & - & - & - \\
3 & - & - & -67.6 & 1.3 \\
4 & 58.3 & 9.9 & - & - \\
5 & 58.5 & 6.5 & -59.6 & 9.9 \\
6 & 58.9 & 6.4 & -62.3 & 7.3 \\
7 & 64.0 & 7.6 & -72.8 & 5.0 \\
8 & - & - & - & - \\
\hline Avg. Deviation* & 2.5 & 5.7 & 11.8 & 1.2 \\
\hline
\end{tabular}

* Note that the data from Event 7 was not used to calculate the average deviation, as this event occurred during southward IMF, causing a high variability in position due to the dynamic environment.

\section{Results for mapping, size, and time travel analysis}

\subsection{Field line mapping using global MHD models}

Table 3 lists the average location between the OpenGGCM and BATS-R-US results and deviation in magnetic latitude and local time (UT) at the Earth's surface for each event. Each footprint location in OpenGGCM and BATS-R-US was obtained by mapping each spacecraft's location into the ionosphere and averaging the four Cluster spacecraft's footprint locations. The deviation in location was calculated by averaging the difference in ionospheric location of the field lines for the starting and ending spacecraft positions and the difference in mapped location between the two models. The ionospheric footprints in the Northern Hemisphere varied from 58.3 degrees to 66.4 degrees magnetic latitude with an average deviation of 2.5 degrees. The magnetic local times in the Northern Hemisphere varied from 6.4 to $9.9 \mathrm{~h}$ with an average deviation of $5.7 \mathrm{~h}$. The ionospheric footprints in the Southern Hemisphere varied from -59.6 degrees to -72.8 degrees magnetic latitude with an average deviation of 11.8 degrees. The magnetic local times in the Southern Hemisphere varied from 1.3 to $9.9 \mathrm{~h}$ with an average deviation 

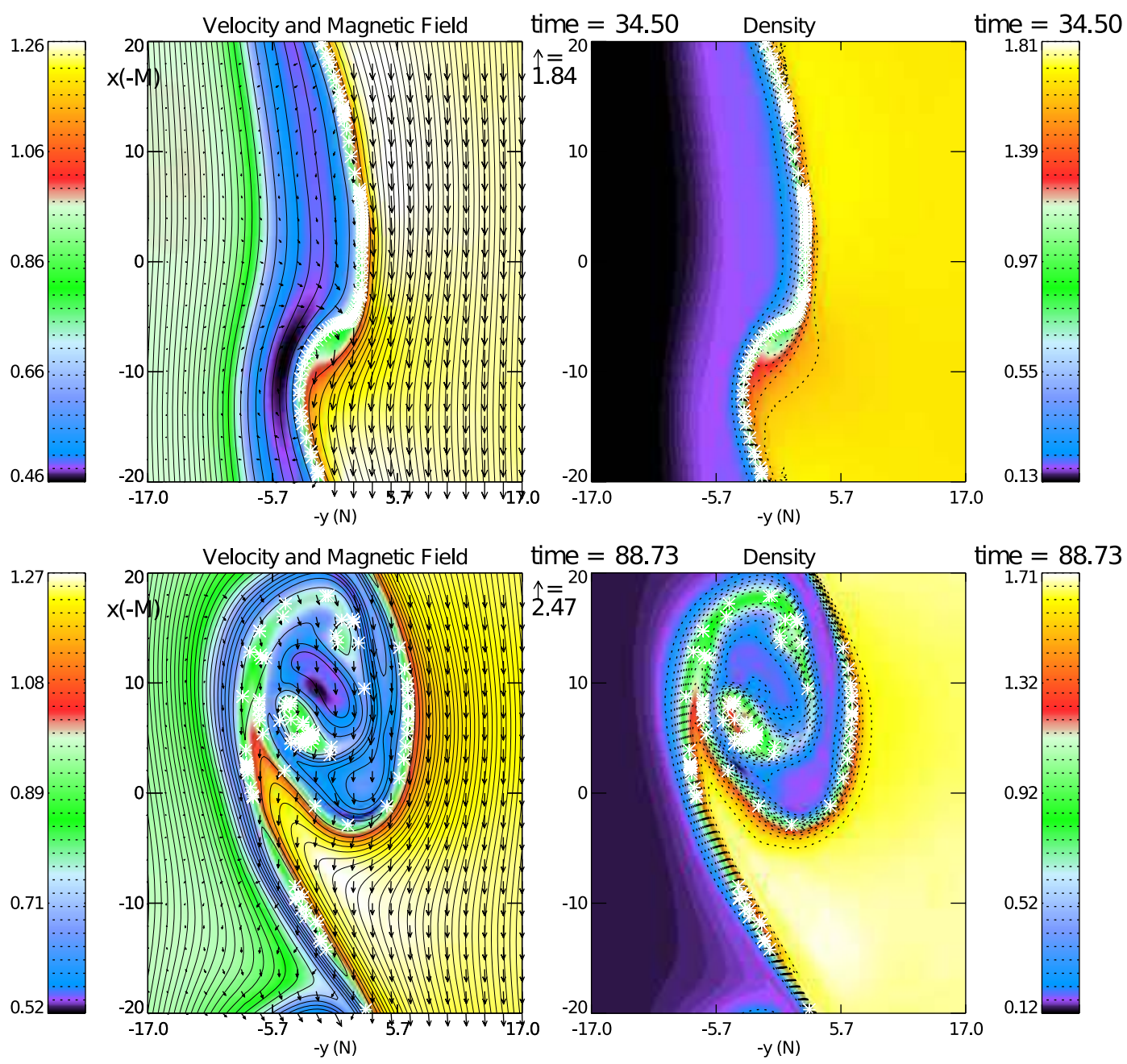

time $=88.73$

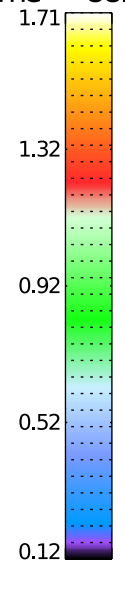

Fig. 7. Example of the onset and evolution of the KHI vortex simulated by local MHD simulations for Event 3. The top- and bottom-left figures show the velocity vectors represented by the arrows and magnetic field depicted by color. The top- and bottom-right figures show the density represented by the color. The two top figures show the onset of the vortex and the bottom two figures represent how the vortex has evolved over time. The asterisks in the figures represent fluid elements. All values listed are in normalized units. The positive $x$ axis is up and the positive $y$ axis is right.

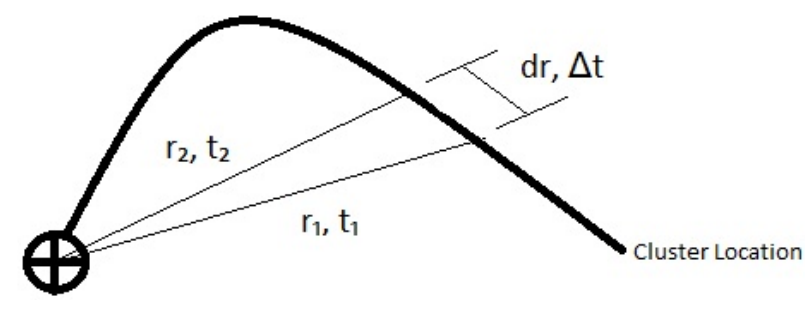

Fig. 8. Illustration of the time lag methodology for calculating the perturbation travel time from the magnetosphere to the ionosphere.

of $1.2 \mathrm{~h}$. Both global MHD models calculated that the KHI which occurred during Event 2 and Event 8 took place on open field lines, that is, field lines which did not connect to the Earth's ionosphere. Both models also calculated that the
KHI which occurred during Event 3 occurred on field lines mapping only to the Southern Hemisphere, whereas the KHI which occurred during Event 4 occurred on field lines mapping only to the Northern Hemisphere.

The events were additionally modeled with BATS-R-US coupled with the Rice Convection Ring Current Model, which coupled the inner and middle magnetosphere with the ionosphere (Toffoletto et al., 2003). The ionospheric footprint locations varied by a difference of 0.1 degrees to 3.68 degrees magnetic latitude and 0.06 to $0.71 \mathrm{~h}$ magnetic local time. These minor location changes are within the deviations of the global models. The BATS-R-US coupled model, however, did map the KHI occurring in Event 4 into the Northern Hemisphere, while the BATS-R-US uncoupled and OpenGGCM models did not. 


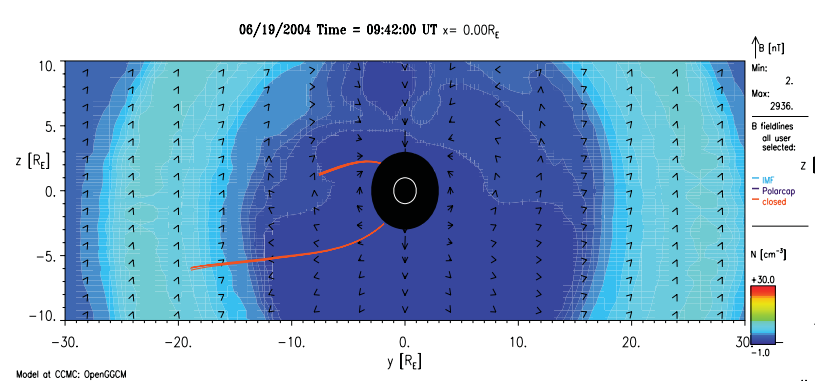

06/19/2004 Time $=09: 42: 00$ UT $y=0.00 R_{E}$
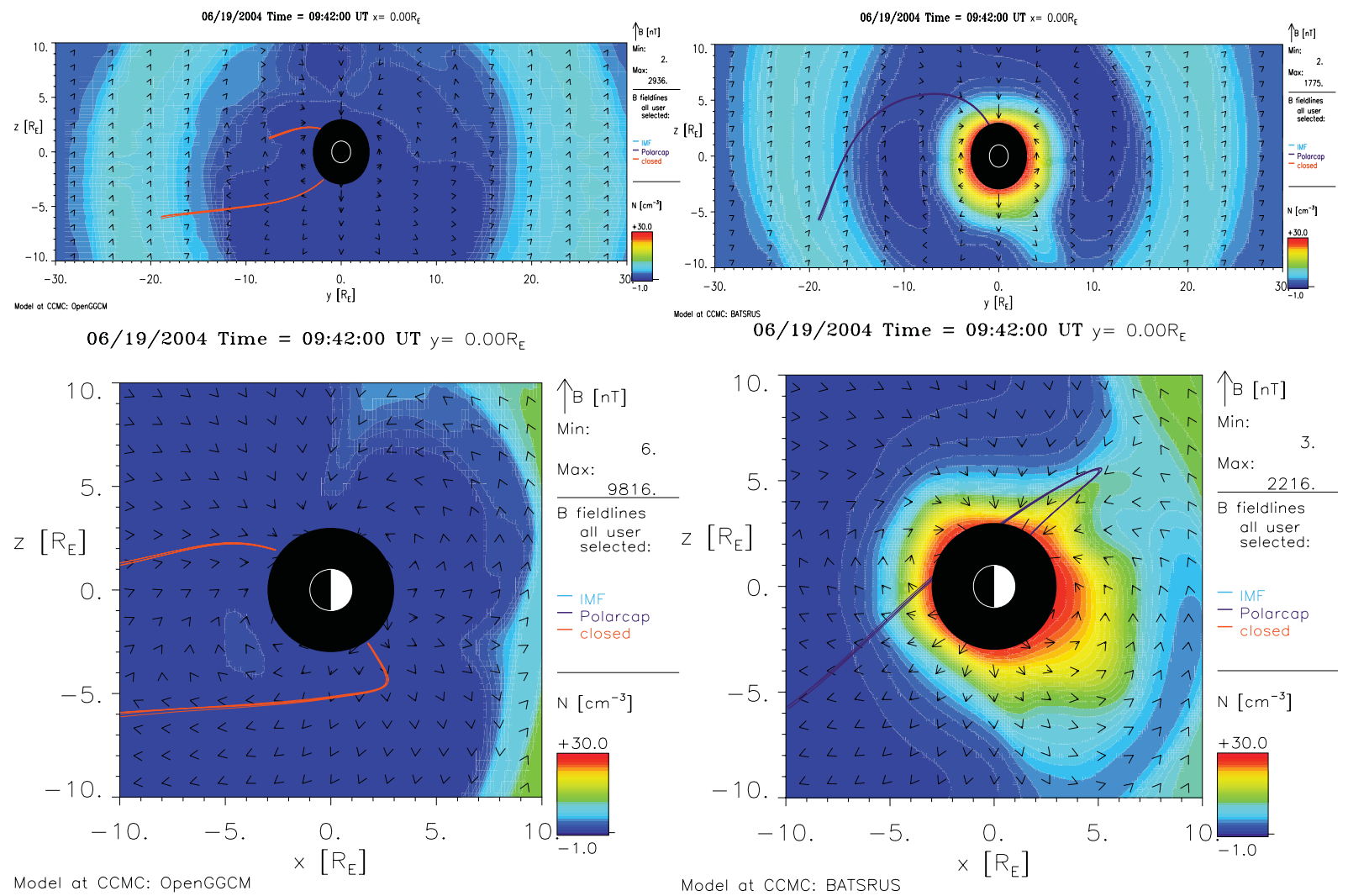

06/19/2004 Time $=09: 42: 00$ UT $y=0.00 R_{E}$

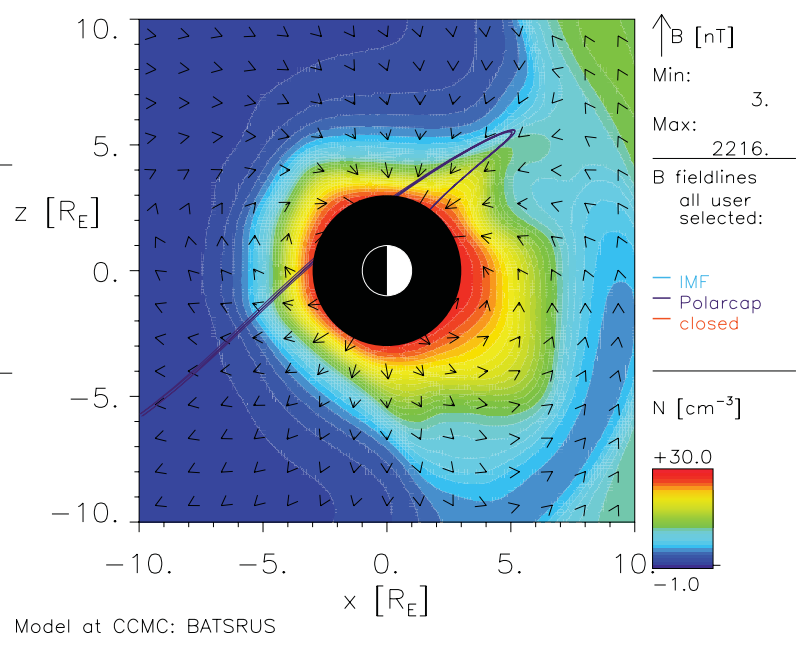

Fig. 9. Difference in the global MHD model's projected field lines which the Cluster constellation encountered through during the KHI for Event 6. The top- and bottom-left images were produced from OpenGGCM and the top- and bottom-right images were produced from BATS-R-US. The spacecraft coordinates can be references from Table 1. Each panel illustrates the magnetic field direction using a black arrow and density using the color bar.

The spatial resolution of the two MHD models was studied to determine if the output frequency of the mapped field lines was significant enough to contribute to the difference in ionospheric location between the two models. While both models were run with identical temporal resolution, OpenGGCM had a higher spatial resolution than BATS-R-US at an average of 191 to $338 \mathrm{~km}$, respectively. A spatial resolution difference is to be expected, as both models utilize different grid sizes and solving scheme, however we do not believe this difference significantly contributed to the footprint difference.

One must also take into account the position of the spacecraft constellation in reference to the vortex. Currently, we assume the spacecraft is located near the center of the vortex. However, if the constellation is located at the edge of the vortex, then one could expect the center of the vortex to be up to half of its size away from the ionospheric footprint location. Using the largest vortex size, this deviation could be up to $300 \mathrm{~km}$ away, corresponding to approximately 2.5 degrees magnetic latitude and $0.2 \mathrm{~h}$ magnetic local time.

\subsection{Field line mapping using TS96 model}

The TS96 field line mapping results are shown in Table 4. The deviation in the location was calculated by averaging the difference in ionospheric location of the field lines for the starting and ending spacecraft positions. The ionospheric footprints in the Northern Hemisphere varied from 72.9 degrees to 80.2 degrees magnetic latitude with an average deviation of 0.07 degrees. The magnetic local times in the Northern Hemisphere varied from 5.0 to $13.8 \mathrm{~h}$ with an average deviation of $0.11 \mathrm{~h}$. The ionospheric footprints in the Southern Hemisphere varied from -72.6 degrees to -83.4 degrees magnetic latitude with an average deviation of 1.9 degrees. The magnetic local times in the Southern Hemisphere varied between 4.9 to $11.9 \mathrm{~h}$ with an average deviation of $-0.2 \mathrm{~h}$. This model calculated that the KHI which occurred during Event 3 occurred on field lines mapping only to the Southern Hemisphere, whereas the KHI which occurred during Event 8 occurred on field lines mapping only to the Northern Hemisphere. These results differ from the global MHD results, as Event 2 mapped into both hemispheres, Event 4 mapped into the Southern Hemisphere, and Event 8 mapped into the Northern Hemisphere. They also greatly differ in 
Table 4. TS96 ionospheric footprint location in magnetic latitude and local time (UT) coordinates. The subindices refer to the mapped field line locations ending in the northern $(\mathrm{N})$ or southern $(\mathrm{S})$ pole.

\begin{tabular}{ccccc}
\hline Event & MLAT $_{\mathrm{N}}$ & MLT $_{\mathrm{N}}$ & MLAT $_{\mathrm{S}}$ & MLT $_{\mathrm{S}}$ \\
\hline 1 & 76.2 & 10.2 & -75.5 & 6.6 \\
2 & 77.2 & 13.8 & -78.8 & 11.9 \\
3 & - & - & -77.7 & 5.3 \\
4 & 75.0 & 6.9 & -72.6 & 4.9 \\
5 & 80.2 & 6.4 & -80.5 & 11.4 \\
6 & 79.6 & 6.1 & -83.4 & 9.1 \\
7 & 74.4 & 5.0 & -76.9 & 5.4 \\
8 & 72.9 & 12.9 & - & - \\
\hline Avg. Deviation & 0.07 & 0.11 & 1.9 & -0.2 \\
\hline
\end{tabular}

the magnetic latitude of the footprint. The magnetic latitude varies between a maximum of -21.07 degrees and minimum of 3.5 degrees, averaging 13.9 degrees of difference between the two types of models. The magnetic local time varies between a maximum of $4.0 \mathrm{~h}$ and minimum of $0.1 \mathrm{~h}$ for both hemispheres, averaging $2.1 \mathrm{~h}$ of difference between the two models. This was the most significant difference between the two different types of models. The difference is perhaps due to the different near-Earth approximations used by the two model types.

\subsection{Ionospheric vorticity area}

Table 5 shows the approximate dimensions of the vortex at $100 \mathrm{~km}$ altitude using $v_{\mathrm{ph}} \approx \frac{1}{2} v_{\mathrm{MSH}}$, resulting in sizes between 62 to $430 \mathrm{~km}$. The vortex size results are within the same order of magnitude as previously published sizes from Lui (1989) and Farrugia et al. (1994) of 50 to $250 \mathrm{~km}$. Deviation from these sizes is on the order of one kilometer per dimension, as a $0.5 R_{E}$ magnetospheric sizing error would only yield a $15 \mathrm{~km}$ error. Table 6 shows the approximate dimensions of the vortex at $100 \mathrm{~km}$ altitude using $v_{\mathrm{ph}} \approx v_{\mathrm{HT}}$, resulting in sizes between 47 to $606 \mathrm{~km}$. These vortex size results are the same order of magnitude as previously published sizes from Lui (1989) and Farrugia et al. (1994) and the results in Table 5. Hasegawa et al. (2004) calculated the scale of one wavelength in the magnetosphere for Event 2 to be between 40000 and $55000 \mathrm{~km}$ using in situ measurements. They inferred the initial thickness of the velocity shear to be roughly 5000 to $7000 \mathrm{~km}$ as the wavelength of the fastest growing $\mathrm{KH}$ mode was estimated to eight times the initial total thickness of the velocity shear. However, Foullon et al. (2008) disagreed with this and determined the wavelength to be between 16000 and $21000 \mathrm{~km}$. Both of these magnetospheric wavelengths are larger than our estimation of 7000 to $12000 \mathrm{~km}$ based on $\lambda=v_{\mathrm{ph}} T$, which suggest that our simple estimation of phase speed using both $v_{\mathrm{ph}} \approx \frac{1}{2} v_{\mathrm{MSH}}$ and $v_{\mathrm{ph}} \approx v_{\mathrm{HT}}$ yields an underestimation. If this trend is also
Table 5. Vortex dimensions for both hemispheres in the ionosphere at $100 \mathrm{~km}$ altitude using $v_{\mathrm{ph}} \approx \frac{1}{2} v_{\mathrm{MSH}}$.

\begin{tabular}{ccccc}
\hline Event & $X[\mathrm{~km}]$ & $Y[\mathrm{~km}]$ & $X[\mathrm{~km}]$ & $Y[\mathrm{~km}]$ \\
\hline & North & North & South & South \\
1 & 258 & 166 & 262 & 168 \\
2 & 108 & 62 & 114 & 66 \\
3 & - & - & 430 & 300 \\
4 & 241 & 225 & - & - \\
5 & 175 & 149 & 186 & 165 \\
6 & 160 & 137 & 169 & 147 \\
\hline
\end{tabular}

valid for other events, our vortex sizes are underestimated at least by factor of approximately $4 / 3$.

\subsection{Perturbation travel time}

Table 7 documents the travel time in seconds from the spacecraft location into an average of $3.6 R_{E}$ altitude at each hemisphere. The difference in travel time duration between the four spacecraft for a given event was under one second on average, therefore the final travel time was represented by the first spacecraft. Travel times varied from $\approx 61 \mathrm{~s}$ to $27 \mathrm{~min} 7 \mathrm{~s}$. All events mapped by OpenGGCM had faster travel times to the Southern Hemisphere. For BATS-R-US, the events which mapped into the Northern Hemisphere had faster travel times, with an exception of Event 3 which only mapped into the Southern Hemisphere. All calculated travel times were within an expected proximity of one another when comparing the results from the two models, except Event 6.

The travel time results from Event 6 shows how the models' results varied due to differences in field line topology. The difference between the BATS-R-US and OpenGGCM times were due to the difference in their projected field line topology, as displayed in Fig. 9. Figure 9 clearly shows the significant topology difference between the two model's magnetic field lines. The two left figures show the field lines in the $Z Y$ and $Z X$ frame from the OpenGGCM model, and the two right figures show identical plots from the BATS-RUS model. OpenGGCM showed a significantly shorter magnetic field line entering the Northern Hemisphere. BATS-RUS modeled a longer and more curved magnetic field line which enters the Northern Hemisphere. An explanation could be that BATS-R-US incorporates a numerical analysis technique where the dipole moment is updated at each iteration, while OpenGGCM does not. One should also take note that Event 5 occurred 20 min before Event 6, and had a travel time half as long in the OpenGGCM results than in the BATS-RUS results. 
Table 6. Vortex dimensions for both hemispheres in the ionosphere at $100 \mathrm{~km}$ altitude using $v_{\mathrm{ph}} \approx v_{\mathrm{HT}}$.

\begin{tabular}{ccccc}
\hline Event & $X[\mathrm{~km}]$ & $Y[\mathrm{~km}]$ & $X[\mathrm{~km}]$ & $Y[\mathrm{~km}]$ \\
\hline & North & North & South & South \\
1 & 226 & 145 & 230 & 147 \\
2 & 82 & 47 & 87 & 50 \\
3 & - & - & 606 & 423 \\
4 & 304 & 285 & - & - \\
5 & 115 & 97 & 121 & 108 \\
6 & 142 & 122 & 150 & 131 \\
\hline
\end{tabular}

\section{Results for ionospheric signatures}

Only events which had available data in their respective ionospheric instruments will be discussed in the following sections. Neither Polar UVI and Image FUV instruments had sufficient data available for analysis during our events.

\subsection{SuperDARN signatures}

Because the radar's reflection region is in the $F$ region, altitudes from 150 to $800 \mathrm{~km}$, new vortex dimensions were calculated. Updated travel times to this altitude were not necessary based on the temporal resolution of the instruments. The vortex dimensions at $600 \mathrm{~km}$ altitude using $v_{\mathrm{ph}} \approx \frac{1}{2} v_{\mathrm{MSH}}$ ranged from 71 to $495 \mathrm{~km}$. The dimensions using the $v_{\mathrm{ph}} \approx$ $v_{\text {HT }}$ method ranged from 55 to $697 \mathrm{~km}$ at $600 \mathrm{~km}$ altitude. SuperDARN's resolution should be sufficient to resolve the vortex in this region.

The coordinate ranges used to observe potential KHI signatures include both global MHD and TS96 ionospheric footprints, as well as their deviations. Table 8 displays the hemisphere, location, speed, size, and vortex type of the observed signatures. In reference to vortex type, type 1 refers to a stationary vortex, type 2 refers to a traveling vortex, and type 3 refers to an event having multiple vortices observed at the same time. The location is categorized as within the TS96 geographic limit, within the MHD geographic limit, or outside both limits. SuperDARN radar covered the ionospheric footprint region for Events 2, 4, 5 and 6 in the Northern Hemisphere and Events 1, 5 and 6 in the Southern Hemisphere. Event 6 did not show any signs of vortices present in the data. Event 1 and 5 located vortices within the TS96 footprint region in the Southern Hemisphere, while the remainder of the events in the Northern Hemisphere located vortices outside both global and TS96 footprint predictions. The vortex speeds varied between 300 and $400 \mathrm{~m} \mathrm{~s}^{-1}$ with sizes between 1000 to $1800 \mathrm{~km}$. These vortex sizes were all larger than the predicted sizes, which ranged approximately from 50 to $600 \mathrm{~km}$. Events 1, 4 and 5 in the Southern Hemisphere were of type 1 , indicating the presence of a single, stationary vortex. Events 2 and 5 were of type 2, indicating the presence of a single vortex which changed locations over time.
Table 7. Travel time in seconds reach approximately $3.6 R_{E}$ towards each hemisphere. $t_{\mathrm{N}}$ and $t_{\mathrm{S}}$ represent travel time to the Northern and Southern Hemisphere, respectively.

\begin{tabular}{ccccc}
\hline Event & $t_{\mathrm{N}}[\mathrm{s}]$ & $t_{\mathrm{S}}[\mathrm{s}]$ & $t_{\mathrm{N}}[\mathrm{s}]$ & $t_{\mathrm{S}}[\mathrm{s}]$ \\
\hline & OpenGGCM & & BATS-R-US & \\
1 & 213.2 & 300 & 103.6 & 566.3 \\
2 & - & - & - & - \\
3 & - & 105.4 & - & 170.4 \\
4 & 108.3 & - & - & - \\
5 & 892.1 & 60.7 & 181.3 & - \\
6 & 1626.7 & 36.9 & 138.9 & - \\
\hline
\end{tabular}

\subsection{SuperMAG signatures}

The coordinate ranges used to observe potential KHI signatures include both global MHD and TS96 ionospheric footprints, as well as their deviations. Table 9 documents the period and change in magnetic field components of the quasiperiodic oscillations at the Cluster spacecraft location. Magnetic oscillations varied up to $[35,25,20] \mathrm{nT}$ in the $X, Y$, and $Z$ directions, respectively. The oscillation periods varied from 1 to $7 \mathrm{~min}$, generally falling within the Pc5 range as suspected by Ohtani et al. (1999). Table 10 documents the signature results from SuperMAG. In the Northern Hemisphere, five events had ground magnetometer stations operating within their footprint range. Events 2, 6 and 7 did not show quasi-periodic magnetic pulsations in their ground data. Event 1 had one station which recorded quasi-periodic magnetic pulsations out of a total one station located within its footprint location. Event 4 had three out of fifteen stations record quasi-periodic magnetic pulsations and Event 5 had six out of thirteen stations record quasi-periodic magnetic pulsations. For the Southern Hemisphere, three events had ground magnetometer stations operating within their footprint range. Event 3 did not record quasi-periodic magnetic pulsations, while one out of one station did record pulsations during Event 5 and one out of five stations record pulsations for Event 6. Overall, magnetic oscillations varied up to [20, $25,7] \mathrm{nT}$ in the $N, E$, and $Z$ directions, respectively. The magnetic field component coordinates $\left(B_{N}, B_{E}, B_{Z}\right)$ refer to the magnetic field pointing in the local magnetic north, local magnetic east, and vertically downward direction. The oscillation periods varied from 4 to $10 \mathrm{~min}$.

The power spectral density (PSD) was calculated along the Pc5 frequency spectrum for the ground and spacecraft magnetometer data. Figure 10 compares the PSD for each Cluster spacecraft's magnetic field components $\left(B_{x}, B_{y}, B_{z}\right)$ and total, as well as the ground magnetometer magnetic field total for Event 1. The total magnetic field PSD was calculated by taking the PSD of each individual component and adding them together. The ground magnetometer magnetic field total adds together the PSD of the $B_{N}, B_{E}$, and $B_{Z}$. The top three frames plot the $x, y$, and $z$ component PSD of the magnetic 
Table 8. Summary of SuperDARN vortices in the Northern and Southern Hemisphere to include footprint location, speed, size, and type. Type 1 refers to a stationary vortex, type 2 refers to a traveling vortex, and type 3 refers to an event having multiple vortices observed at the same time.

\begin{tabular}{cccccc}
\hline Event & Hemisphere & Location & Speed $\left[\mathrm{m} \mathrm{s}^{-1}\right]$ & Size $[\mathrm{km}]$ & Type \\
\hline 1 & South & In TS96 limit & 400 & 1000 & 1 \\
2 & North & Outside limit & 300 & 1100 & 2 \\
4 & North & Outside limit & 300 & 1200 & 1 \\
5 & North & Outside limit & 400 & 1800 & 2 \\
& South & In TS96 limit & 300 & 1200 & 1 \\
6 & North & - & - & - & - \\
& South & - & - & - & - \\
\hline
\end{tabular}

Table 9. Change in magnetic field components taken at the Cluster location for each event and the period of the oscillations.

\begin{tabular}{ccc}
\hline Event & $\Delta B_{x}, \Delta B_{y}, \Delta B_{z}[\mathrm{nT}]$ & Period $[\mathrm{m}]$ \\
\hline 1 & $35,10,12$ & $4-7$ \\
2 & $12,17,12$ & $3.5-4$ \\
3 & $7,12,13$ & $2.5-3$ \\
4 & $17,7,20$ & $3-5$ \\
5 & $10,5,7$ & $3.5-4$ \\
6 & $20,12,7$ & $2.5-3$ \\
7 & $25,25,20$ & $1-3.5$ \\
\hline
\end{tabular}

Table 10. Summary of SuperMAG clear quasi-periodic oscillations in the Northern and Southern Hemisphere to include station acronym, change in magnetic field, and period. The asterisk refers to stations in the Southern Hemisphere. Station (Total) refers to the station name which displayed results and the total number of SuperMAG stations that were within the geographic range of our event. Only those stations which provided clear quasi-periodic oscillations are documented.

\begin{tabular}{lccc}
\hline Event & Station (Total) & $\Delta B_{\mathrm{N}}, \Delta B_{E}, \Delta B_{Z}[\mathrm{nT}]$ & Period $[\mathrm{m}]$ \\
\hline 1 & VIZ (1) & $15,25,5$ & 7 \\
$3^{*}$ & $-(1)$ & - & - \\
2 & $-(4)$ & - & - \\
4 & IGC (15) & $0,10,0$ & 7 \\
& IQA & $0,15,3$ & 7 \\
& PGC & $3,10,0$ & $6-7$ \\
5 & ATU (13) & $0,5,5$ & $5-7$ \\
& GHB & $15,0,5$ & $6-8$ \\
& KUV & $3,3,0$ & $6-7$ \\
& NAQ & $5,3,5$ & 6 \\
& SKT & $20,0,7$ & $6-7$ \\
& STF & $20,10,7$ & $6-7$ \\
$5^{*}$ & MAW (1) & $0,10,0$ & $7-10$ \\
6 & $-(1)$ & - & - \\
$6^{*}$ & B15 (5) & $0,13,0$ & $4-5$ \\
7 & $-(6)$ & - & - \\
$7^{*}$ & $-(1)$ & - & - \\
\hline
\end{tabular}

Table 11. Comparison between the dominating frequencies in Cluster and SuperMAG magnetic field data. The dominating frequencies are those which had the higher PSD when compared to the surrounding frequencies.

\begin{tabular}{ccc}
\hline Event & Instrument & Dominating Freq. [mHz] \\
\hline 1 & Cluster & $3.1,3.6$ \\
& VIZ & $2.2,2.5,3.0,3.6,3.8$ \\
4 & Cluster & $4.2,5.0,6.7,9.2$ \\
& IGC & $3.2,5.6,7.9$ \\
& IQA & $3.2,5.6,7.9$ \\
& PGC & $3.2,5.6,7.9$ \\
5 & Cluster & $2.8,5.0,5.7$ \\
& MAW & 2.0 \\
& SKT & $2.7,4.7$ \\
& STF & $2.7,4.7$ \\
& ATU & $2.7,6.0$ \\
& GHB & $2.0,2.7,4.7$ \\
& KUV & $2.0,2.7,5.3$ \\
6 & NAQ & $2.7,3.3,5.3$ \\
& Cluster & $4.1,7.4$ \\
& B15 & $4.0,6.3$ \\
\hline
\end{tabular}

field as recorded by Cluster. The fourth frame plots the total magnetic field PSD from Cluster, and the fifth frame plots the total magnetic field PSD from the VIZ ground magnetometer station. The three highlighted columns through each plot represent the three dominating peaks in the frequency range. As the first and last columns consist of two frequencies, five frequencies are present in the ground data which have the highest PSD throughout the Pc5 frequency spectra. The Cluster PSD data reveals two dominating frequencies, 3.1 and $3.6 \mathrm{mHz}$. Both of these frequencies are present in the VIZ data within $0.1 \mathrm{mHz}$.

This analysis was carried out for each event which indicated quasi-periodic pulsations in their ground instruments and was documented in Table 11. The dominating frequencies indicated for the Cluster and ground data are those frequencies which had a higher PSD in their total magnetic field than the surrounding regions. Station VIZ recorded five different dominating frequencies during Event 1, two of which 


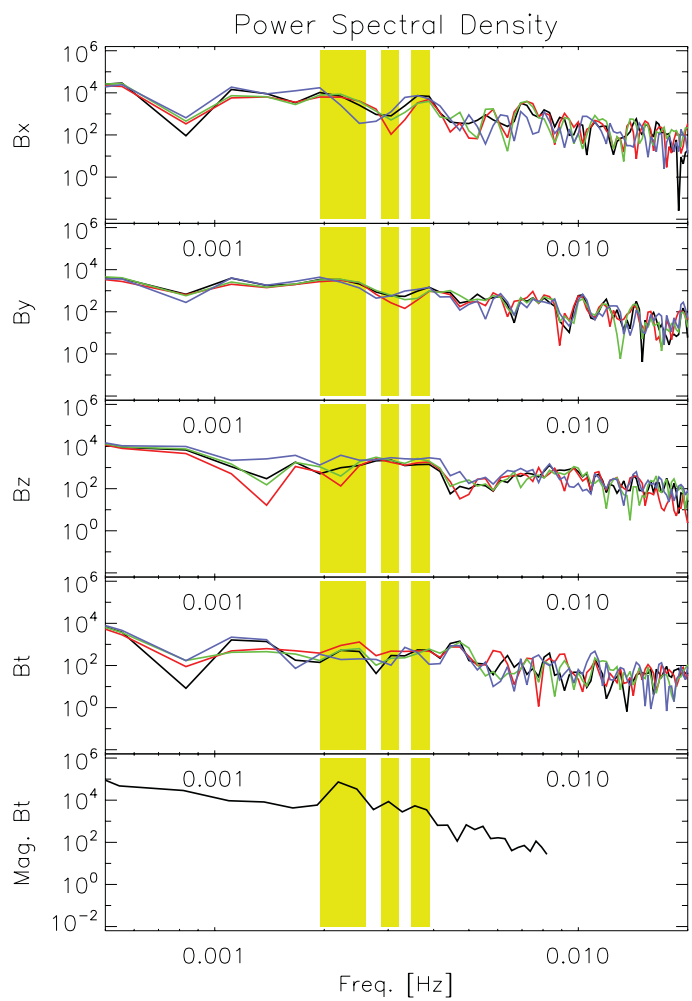

Fig. 10. PSD $\left[\mathrm{nT}^{2} \mathrm{~Hz}^{-1}\right.$ ] over the Pc5 frequency range for Event 1 from 05:00 to 06:00 UT. The panels represent the PSD of the Cluster magnetic field components and total magnetic field, as well as the ground station total magnetic field, respectively. In order, the four spacecrafts are represented in the top four panels by black, red, green, and blue colors. The dominating frequencies in the ground magnetometer data are highlighted in yellow throughout the figure. The frequency range only includes those in the Pc5 spectrum.

were within $0.1 \mathrm{mHz}$ of the identified Cluster-dominating frequencies. Stations IGC, IQA, and PGC recorded three dominating frequencies, all identical. One frequency was within $0.6 \mathrm{mHz}$ of the four Cluster dominating frequencies. During Event 5, six out of seven ground stations recorded a dominating frequency within $0.1 \mathrm{mHz}$ of the three Cluster frequencies. Five of the seven stations also recorded frequencies within $0.3 \mathrm{mHz}$ of another dominating Cluster frequency. Station B15 recorded two different frequencies during Event 6, one of which was within $0.1 \mathrm{mHz}$ of the two Cluster dominating frequencies. Events 1, 5, and 6 were similar to the previously published results obtained by Ohtani et al. (1999) whom discovered dominating frequencies within $0.2 \mathrm{mHz}$ of the spacecraft frequency. All oscillation frequencies were higher in the ground magnetometer data than the Cluster data.

\section{Conclusions and discussion}

Establishing a robust identification method of the ionospheric signatures resulting from magnetospheric KHI would be important, as it would allow scientists to reverse engineer the process in order to locate a magnetospheric KHI event using ionospheric data. As the KHI can generate FACs, it can modify the ionospheric conductivities and hence the dynamics of the magnetosphere-ionosphere coupling which can lead to local and global changes of this system. Reconnection initiated by KHI can also create a parallel electric field, causing an acceleration of particles along the magnetic field. Also, pitch angle scattering into the loss cone produced by reconnection in the vortices may lead to particle precipitation into the atmosphere. In the present paper we have determined an ionospheric location, size, and perturbation travel time from magnetosphere to the ionosphere of nine events of quasi-periodic oscillations at the flank magnetopause that exhibited KHI-like signatures. We then looked for the potential ionospheric signatures of these events. The conclusions are as follows:

- The mapped field lines produced from the global MHD models ranged in ionospheric position from a magnetic latitude of 58.3 degrees to 66.4 degrees in the Northern Hemisphere and -59.6 degrees to -72.8 degrees in the Southern Hemisphere. The ionospheric magnetic local time ranged between 6.4 to $9.9 \mathrm{~h}$ in the Northern Hemisphere and 1.3 to $9.9 \mathrm{~h}$ in the Southern Hemisphere. These magnetic latitudes were less than the latitudes at which Lui (1989) and Farrugia et al. (1994) observed their auroral bright spots and where McHenry et al. (1990) observed traveling convection vortices. The mapped magnetic latitude range was similar to where Ohtani et al. (1999) observed the Pc5 magnetometer pulsations.

- The mapped field lines produced from the TS96 model ranged in ionospheric position from a magnetic latitude from 72.9 degrees to 80.2 degrees in the Northern Hemisphere and -72.6 degrees to -83.4 degrees in the Southern Hemisphere. The ionospheric magnetic local time ranged between 5.0 to $13.8 \mathrm{~h}$ in the Northern Hemisphere and 4.9 to $11.9 \mathrm{~h}$ in the Southern Hemisphere. These magnetic latitudes were similar to the latitudes at which Lui (1989) and Farrugia et al. (1994) observed their auroral bright spots, where Ohtani et al. (1999) observed the Pc5 magnetometer pulsations, and where McHenry et al. (1990) observed traveling convection vortices. They were also mapped to significantly higher magnetic latitudes than the global MHD models.

- Typical Alfvén wave travel time from spacecraft location to the closest ionosphere ranged between 0.6 to $3.6 \mathrm{~min}$. 
- The projected ionospheric size calculated at an altitude of $100 \mathrm{~km}$ ranged from 47 to $606 \mathrm{~km}$, the same order of magnitude as previously determined potential ionospheric signature sizes measured by Lui (1989) and Farrugia et al. (1994).

- Stationary and traveling convection vortices were observed in the SuperDARN data during Events 2, 4 and 5 in the Northern Hemisphere and Events 1 and 5 in the Southern Hemisphere. All vortices sizes were of 1000 and $1800 \mathrm{~km}$ in size, traveling at speeds between 300 and $400 \mathrm{~m} \mathrm{~s}^{-1}$. Events 1, 4 and 5 had a single stationary vortex present and Events 2 and 5 had a single traveling convection vortex present. Only the observed vortices for Events 1 and 5 were within our estimated footprint.

- Pc5 magnetic oscillations were observed in the SuperMAG data during Events 1, 4 and 5 in the Northern Hemisphere and Events 5 and 6 in the Southern Hemisphere. The oscillations had periods of 4 and $10 \mathrm{~min}$ with amplitudes of 3 to $25 \mathrm{nT}$. They were all located within the ionospheric footprint range. These observations were consistent with previously published ground magnetometer signatures studied by Ohtani et al. (1999) and the observed periodicity of the KHI occurring at the LLBL. The ground magnetometer data for Events 1, 5 and 6 had PSD peaks at frequencies that were within $0.1 \mathrm{mHz}$ of the peaks found in the corresponding Cluster data. These observations were consistent with those from Ohtani et al. (1999), whom observed Pc5 frequencies in spacecraft and ground data within $0.2 \mathrm{mHz}$ of one another.

We believe our methods for field line mapping into the ionosphere were successful at providing a general observational region for signatures, but not an accurate location for each event. Footprint accuracy showed its difficulty particularly using SuperDARN data. Determining whether a vortex in the SuperDARN data was due to KHI or background convection pattern may be easier to differentiate with better mapping accuracy. Generally, it is believed that southward IMF causes a two-cell convection pattern to arise in the polar caps, while northward IMF causes a four-cell convection pattern. However, much research continues on the subject as multiple cells can arise in either pattern, as well as distorted or wrapped versions of the "standard" patterns (Knipp et al., 1991). IMF plays a large role when determining the background convection pattern. While convection vortices may be visible in the data, northern IMF orientation makes it difficult for one to conclude whether this KHI induced signature was actually a convection cell from a four-cell convection pattern or other multi-cell pattern. Knipp et al. (1991) noted that multiple convection cells can arise particularly during transitions between the two IMF orientations. Ruohoniemi and Greenwald (1998) documented a 2 min change in convection cell direction during a northward to southward IMF transition which occurred over $2.5 \mathrm{~min}$. Differentiating between the typical background convection patterns and our expected vortex signature thus becomes difficult. More extensive work is necessary to overcome this observational challenge. The $F$ region is dynamic and our research would benefit from a more accurate footprint. This may allow one to differentiate between ionospheric anomalies produced by KHI versus a vortex due to a two-cell or four-cell convection pattern.

Running high-resolution models could provide more accurate ionospheric footprint results. It would be desirable to simulate these events with very high resolution (40 to $100 \mathrm{~km}$ ) in global models to resolve both cusp, day-side and flank KHI regions simultaneously. This would allow one to address the question of whether the quasi-periodic oscillations at the flank could be produced by a flux transfer event originating from the day-side or cusp for these conditions or what the combined ionospheric signatures would look like if both processes occurred simultaneously.

It would also be important to determine the exact cause of the large discrepancy in mapped ionospheric footprint location in magnetic field latitude between the TS96 and global MHD models. Both model types located the footprint to the same altitude, however there is up to a 20 degree difference in magnetic latitude. Due to its semi-empirical nature, the TS96 model inherently includes the effects of the kinetic physics, while the global MHD models did not (with the partial exception of the BATS-R-US ring current model version, which was ran as a special case in our research). However, the global MHD models require more environmental parameters to be defined than the TS96 model, which only requires the disturbance solar time index, solar wind dynamic pressure and velocity (1-D) and the $B_{y}$ and $B_{x}$ component of the IMF to run. The global models utilize an electric potential solver and dipole approximation while TS96 uses the IGRF-2011 model coefficients for the ionosphere to map ionospheric footprints to the Earth's surface. The high order terms in the spherical harmonic expansion of the IGRF2011 coefficients overtake the dipole approximation term in the expansion as one nears the Earth's surface. Therefore, the IGRF-2011 model should be a more accurate model for near-Earth approximations than a dipole model. If the global models could include the option to map the near-Earth environment using IGRF-2011, this could lead to more accurate mapping results.

Acknowledgements. This research was performed during E. Dougal's undergraduate and graduate studies at Embry-Riddle Aeronautical University. E. Dougal's, K. Nykyri's and T. Moore's research is funded by National Science Foundation Grant 0847120. Global MHD simulations have been provided by the Community Coordinated Modeling Center at Goddard Space Flight Center through their public runs on request system (http://ccmc.gsfc.nasa.gov). The CCMC is a multi-agency partnership between NASA, AFMC, AFOSR, AFRL, AFWA, NOAA, NSF and ONR. The OpenGGCM 
Model was developed by Joachim Raeder at University of New Hampshire and Timothy Fuller-Rowell at NOAA. The BATS-R-US Model was developed by Tamas Gombosi et al. at the University of Michigan. The TS89 4D Orbit Viewer software was provided by NASA Goddard Space Flight Center Space Physics Data Facility, plotted with TIPSOD Version 3.1.28. The original 2-D MHD code was developed by A. Otto. We acknowledge all Cluster instrument teams and the ESA Cluster Active Archive for providing processed science-level data for download. We acknowledge NASA Goddard Space Flight Center Satellite Situation Center Web for providing coordinate system transformations.

Topical Editor L. Blomberg thanks two anonymous referees for their help in evaluating this paper.

\section{References}

Balogh, A., Dunlop, M. W., Cowley, S. W. H., Southwood, D. J., Thomlinson, J. G., and magnetometer team, C.: The Cluster Magnetic Field Investigation, Space Sci. Rev., 79, 65-91, 1997.

Balogh, A., Carr, C. M., Acuña, M. H., Dunlop, M. W., Beek, T. J., Brown, P., Fornacon, K.-H., Georgescu, E., Glassmeier, K.H., Harris, J., Musmann, G., Oddy, T., and Schwingenschuh, K.: The Cluster Magnetic Field Investigation: overview of in-flight performance and initial results, Ann. Geophys., 19, 1207-1217, doi:10.5194/angeo-19-1207-2001, 2001.

Bisikalo, D. V., Shematovich, V. I., Gérard, J.-C., Meurant, M., Mende, S. B., and Frey, H. U.: Remote sensing of the proton aurora characteristics from IMAGE-FUV, Ann. Geophys., 21, 2165-2173, doi:10.5194/angeo-21-2165-2003, 2003.

Boardsen, S., Sundberg, T., Slavin, J., Anderson, B., Korth, H., Solomon, S., and Blomberg, L.: Observations of KelvinHelmholtz waves along the dusk-side boundary of Mercury's magnetosphere during MESSENGER's third flyby, Geophys. Res. Lett., 115, 2066-2074, 2010.

Claudepierre, S. G., Elkington, S. R., and Wiltberger, M.: Solar wind driving of magnetospheric ULF waves: Pulsations driven by velocity shear at the magnetopause, J. Geophys. Res.-Space, 113, A05218, doi:10.1029/2007JA012890, 2008.

Deehr, C. S., Rees, M. H., Belon, A. E. H., Romick, G. J., and Lummerzheim, D.: Influence of the ionosphere on the altitude of discrete auroral arcs, Ann. Geophys., 23, 759-766, doi:10.5194/angeo-23-759-2005, 2005.

Fairfield, D., Otto, A., Mukai, T., Kokubun, S., Lepping, R., Steinberg, J., Lazarus, A., and Tamamoto, T.: Geotail observations of the Kelvin-Helmholtz instability at the equatorial magnetotail boundary for parallel northward fields, J. Geophys. Res., 2115921173, doi:10.1029/1999JA000316, 2000.

Fairfield, D. H., Kuznetsova, M. M., Mukai, T., Nagai, T., Gombosi, T. I., and Ridley, A. J.: Waves on the dusk flank boundary layer during very northward interplanetary magnetic field conditions: Observations and simulation, J. Geophys. Res.-Space, 112, A8206, doi:10.1029/2006JA012052, 2007.

Farrugia, C., Sandholt, P., and Burlaga, L.: Auroral activity associated with Kelvin-Helmholtz instability at the inner edge of the low-latitude boundary layer, J. Geophys. Res., 99, 19403-19411, 1994.

Finlay, C., Maus, S., Beggan, C., Bondar, T., Chambodut, A., Chernova, T., Chulliat, A., Golovkov, V., Hamilton, B., Hamoudi, A., Holme, R., Hulot, G., Kuang, W., Langlais, B., Lesur, V., Lowes,
F., Luhr, H., Macmillan, S., Mandea, M., McLean, S., Manoj, C., Menvielle, M., Michaelis, I., Olsen, N., Rauberg, J., Rother, M., Sabaka, T., Tangborn, A., Toffner-Clausen, L., Thebault, E., Thomson, A., Wardinski, I., Wei, Z., and Zvereva, T.: International Geomagnetic Reference Field: the eleventh generation. International Association of Geomagnetism and Aeronomy, Working Group V-MOD, Geophys. J. Int., 183, 1216-1230, 2010.

Foullon, C., Farrugia, C., Fazakerley, A., Owen, C., Gratton, F., and Torbert, R.: Evolution of Kelvin-Helmholtz activity on the dusk flank magnetopause, J. Geophys. Res., 113, A11203, doi:10.1029/2008JA013175, 2008.

Gjerloev, J.: A Global Ground-Based Magnetometer Initiative, Eos, Transactions American Geophysical Union, 90, 230-231, 2009.

Gombosi, T., Toth, G., Zeeuw, D., Hansen, K., Kabin, K., and Powell, K.: Semirelativistic magnetohydrodynamics and physicsbased convergence acceleration, J. Comput. Phys., 177, 176-205, 2002.

Gombosi, T., Powell, K., Zeeuw, D., Clauer, C., Hansen, K., Manchester, W., Ridley, A., Roussev, I., Sokolov, I., and Stout, Q.: Solution-adaptive magnetohydrodynamics for space plasmas: Sun-to-Earth simulations, Comput. Sci. Eng., 6, 14-35, 2004.

Greenwald, R., Baker, K., Dudeney, J., Pinnock, M., Jones, T., Thomas, E., Villain, J., Cerisier, J., Senior, C., Hanuise, C., Hunsucker, R., Sofko, G., Koehler, J., Nielsen, E., Pellinen, R., Walker, A. D. M., Sato, N., and Tamagishi, H.: A global view of the dynamics of high-latitude convection, Space Sci. Rev., 71, 761-796, 1995.

Guo, X. C., Wang, C., and Hu, Y. Q.: Global MHD simulation of the Kelvin-Helmholtz instability at the magnetopause for northward interplanetary magnetic field, J. Geophys. Res.-Space, 115, A10218, doi:10.1029/2009JA015193, 2010.

Hasegawa, H., Fujimoto, M., Phan, T., Reme, H., Balogh, A., Dunlop, M., Hashimoto, C., and TanDokoro, R.: Transport of solar wind into Earth's magnetosphere through rolled-up KelvinHelmholtz vortices, Nature, 430, 755-758, 2004.

Hasegawa, H., Retino, A., Vaivads, A., Khotyanintsev, Y., Andre, M., Nakamura, T., Teh, W., Sonnerup, B., Schwartz, S., Seki, Y., Fujimoto, M., Saito, Y., Reme, H., and Canu, P.: KelvinHelmholtz waves at the Earth's magnetopause: Multiscale development and associated reconnection, J. Geophys. Res., 114, A12207, doi:10.1029/2009JA014042, 2009.

Hwang, K.-J., Kuznetsova, M., Sahraoui, F., Goldstein, M., Lee, E., and Parks, G.: Kelvin-Helmholtz waves under southward interplnetary magnetic field, J. Geophys. Res., 116, A08210, doi:10.1029/2011JA016596, 2011.

Knipp, D., Richmond, A., Emery, B., Crooker, N., de La Beaujardiere, O., Evans, D., and Kroehl, H.: Ionospheric convection response to changing IMF direction, Geophys. Res. Lett., 18, 721-724, 1991.

Li, W. Y., Guo, X. C., and Wang, C.: Spatial distribution of KelvinHelmholtz instability at low-latitude boundary layer under different solar wind speed conditions, J. Geophys. Res.-Space, 117, A08230, doi:10.1029/2012JA017780, 2012.

Lui, A.: Auroral Bright Spots on the Dayside Oval, J. Geophys. Res., 94, 5515-5522, 1989.

Masters, A., Achilleos, N., Kivelson, M., Sergis, N., Dougherty, M., Thomsen, M., Arridge, C., Krimigis, S., McAndrews, H., Kanani, S., Krupp, N., and Coates, A.: Cassini observations of 
a Kelvin-Helmholtz vortex in Saturn's outer magnetosphere, J. Geophys. Res., 115, 1769, doi:10.1029/2010JA015351, 2010.

McHenry, M., Clauer, C., Friis-Christensen, E., Newell, P., and Kelly, J.: Ground Observations of Magnetospheric Boundary Layer Phenomena, J. Geophys. Res., 95, 14995-15005, doi:10.1029/JA095iA09p14995, 1990.

Miura, A. and Pritchett, P.: Nonlocal stability analysis of the MHD Kelvin-Helmholtz instability in a compressible plasma, J. Geophys. Res., 87, 7431, doi:10.1029/JA087iA09p07431, 1982.

Moore, T.: Identifying Signatures of Plasma Waves and Reconnection Associated with Kelvin-Helmholtz Instabilities, Master's thesis, Embry-Riddle Aeronautical University, 2012.

Nykyri, K.: Influence of the Kelvin-Helmholtz instability on the plasma transport at the magnetospheric boundary, $\mathrm{Ph} . \mathrm{D}$. thesis, University of Alaska, Fairbanks, 2003.

Nykyri, K. and Otto, A.: Plasma transport at the magnetospheric boundary due to reconnection in Kelvin-Helmholtz vortices, Geophys. Res., 28, 3565-3568, 2001.

Nykyri, K., Otto, A., Lavraud, B., Mouikis, C., Kistler, L. M., Balogh, A., and Rème, H.: Cluster observations of reconnection due to the Kelvin-Helmholtz instability at the dawnside magnetospheric flank, Ann. Geophys., 24, 2619-2643, doi:10.5194/angeo-24-2619-2006, 2006.

Ohtani, S., Rostoker, G., Takahashi, K., Angelopoulos, V., Nakamura, M., Waters, C., Singer, H., Kokubun, S., Tsuruda, K., Hughes, W., Potemra, T., Zanetti, L., Gary, J., Lui, A., and Williams, D.: Coordinated ISTP satellite and ground observations of morningside Pc5 waves, J. Geophys. Res., 104, 23812397, doi:10.1029/1998JA900037, 1999.

Otto, A.: 3D resistive MHD computations of magnetospheric physics, Computational Physics Communications, 1990.

Otto, A. and Fairfield, D.: Kelvin-Helmholtz instability at the magnetotail boundary: MHD simulation and comparison with Geotail observations, J. Geophys. Res., 105, 21175-21190, doi:10.1029/1999JA000312, 2000.

Potter, D.: Computational Physics, John Wiley, 1973.

Powell, K., Roe, P., Linde, T., Jombosi, J., and Zeeuw, D.: A solution-adaptive upwind scheme for ideal magnetohydrodynamics, J. Comput. Phys., 154, 284-309, 1999.

Raeder, J., Wang, Y., and Fuller-Rowell, T.: Geomagnetic Storm Simulation with a Coupled Magnetospheric-IonosphericThermospheric Model, Geophys. Monogr. Ser., 125, 377-384, 2001.

Rème, H., Aoustin, C., Bosqued, J. M., Dandouras, I., Lavraud, B., Sauvaud, J. A., Barthe, A., Bouyssou, J., Camus, Th., Coeur-Joly, O., Cros, A., Cuvilo, J., Ducay, F., Garbarowitz, Y., Medale, J. L., Penou, E., Perrier, H., Romefort, D., Rouzaud, J., Vallat, C., Alcaydé, D., Jacquey, C., Mazelle, C., d’Uston, C., Möbius, E., Kistler, L. M., Crocker, K., Granoff, M., Mouikis, C., Popecki, M., Vosbury, M., Klecker, B., Hovestadt, D., Kucharek, H., Kuenneth, E., Paschmann, G., Scholer, M., Sckopke, N., Seidenschwang, E., Carlson, C. W., Curtis, D. W., Ingraham, C., Lin, R. P., McFadden, J. P., Parks, G. K., Phan, T., Formisano, V., Amata, E., Bavassano-Cattaneo, M. B., Baldetti, P., Bruno, R., Chionchio, G., Di Lellis, A., Marcucci, M. F., Pallocchia, G., Korth, A., Daly, P. W., Graeve, B., Rosenbauer, H., Vasyliunas, V., McCarthy, M., Wilber, M., Eliasson, L., Lundin, R., Olsen, S., Shelley, E. G., Fuselier, S., Ghielmetti, A. G., Lennartsson, W., Escoubet, C. P., Balsiger, H., Friedel, R., Cao, J.-B., Kovrazhkin, R.
A., Papamastorakis, I., Pellat, R., Scudder, J., and Sonnerup, B.: First multispacecraft ion measurements in and near the Earth's magnetosphere with the identical Cluster ion spectrometry (CIS) experiment, Ann. Geophys., 19, 1303-1354, doi:10.5194/angeo19-1303-2001, 2001.

Ruohoniemi, J. and Greenwald, R.: The response of high-latitude convection to a sudden southward IMF turning, Geophys. Res. Lett., 25, 2913-2916, 1998.

Sonnerup, B. U. Ö., Paschmann, G., and Phan, T.-D.: Fluid aspects of reconnection at the magnetopause, In situ observations, p. 167, 1995.

Sundberg, T., Boardsen, S., Slavin, J., Blomberg, L., and Korth, H.: The Kelvin-Helmholtz instability at Mercury: An assessment, Planet. Space Sci., 58, 1434-1441, 2010.

Terada, N., Machida, S., and Shinagawa, H.: Global hybrid simulation of the Kelvin-Helmholtz instability at the Venus ionopause, J. Geophys. Res., 107, 1471-1490, 2002.

Toffoletto, F., Sazykin, S., Spiro, R., and Wolf, R.: Inner magnetospheric modeling with the Rice Convection Model, Space Sci. Rev., WISER special issue, 108, 175-196, 2003.

Tóth, G., Van der Holst, B., Sokolov, I. V., De Zeeuw, D. L., Gombosi, T. I., Fang, F., Manchester, W. B., Meng, X., Najib, D. Powell, K. G., Stout, Q. F., Glocer, A., Ma, Y.-J., and Opher, M.: Adaptive numerical algorithms in space weather modeling, J. Comput. Phys., 231, 870-903, 2012.

Tsyganenko, N., Model, M. F., and Routines, F.: GEOPACK-2008, NASA GSFC, 2008.

Tsyganenko, N. A. and Stern, D. P.: Modeling the global magnetic field of the large-scale Birkeland current systems, J. Geophys. Res., 101, 27187-27198, 1996.

Wing, S., Johnson, J., Newell, P., and Meng, C.-I.: Dawn-dusk asymmetries, ion spectra, and sources in the northward interplanetary magnetic field plasma sheet, J. Geophys. Res.-Space, 110, A08205, doi:10.1029/2005JA011086, 2005.

Wolff, R., Goldstein, B., and Yeates, C.: The Onset and Development of Kelvin-Helmholtz Instability at the Venus Ionopause, J. Geophys. Res., 85, 7697-7707, 1980. 